\title{
Modeling the Inhomogeneous Response and Formation of Shear Bands in Steady and Transient Flows of Entangled Liquids
}

\author{
Lin Zhou, Paula A. Vasquez, L. Pamela Cook, Gareth H. McKinley ${ }^{\dagger}$
}

July 30, 2007

\begin{abstract}
We simulate the spatial and temporal evolution of inhomogeneous flow fields in viscometric devices such as cylindrical Couette cells. The computations focus on a class of two species elastic network models which are prototypes for a model which can capture, in a self-consistent manner, the creation and destruction of elastically-active network segments as well as diffusive coupling between the microstructural conformations and the local state of stress in regions with large spatial gradients of local deformation. For each of these models, the 'flow curve' of stress and apparent shear rate resulting from an assumption of homogeneous deformation is non-monotonic and linear stability analysis shows that the region of non-monotonic response is unstable. Steady-state calculations of the full inhomogeneous flow field lead to localized shear bands that grow linearly in extent across the gap as the apparent shear rate is incremented. Time-dependent calculations in step strain experiments and in start up of steady shear flow show that the velocity profile in the gap and the total stress measured at the bounding surfaces are coupled and evolve in a complex non-monotonic manner as the shear bands develop. These spatio-temporal dynamics are consistent with time-resolved particle imaging velocimetry measurements in both concentrated solutions of monodisperse entangled polymers and in wormlike micellar solutions. The computational results have a number of implications for experimental observations of 'apparent' or 'gap-averaged' quantities in nearly-viscometric devices, and lead to plateaus or 'yield-like' transitions in the steady flow curve and deviations from the Lodge-Meissner relation in non-ideal step shearing deformations.
\end{abstract}

\footnotetext{
* Department of Mathematical Sciences, University of Delaware
}

${ }^{\dagger}$ Department of Machanical Engineering, MIT 


\section{Introduction}

A central assumption behind rheometry is that the velocity field generated in a rheometer remains viscometric at all deformation rates. By judicious choice of geometric parameters such as the cone angle or gap separation characterizing the test fixtures, any spatial inhomogeneities are expected to remain small. However, many complex fluids including concentrated solutions of wormlike micelles and highly monodisperse entangled polymer solutions undergo a more dramatic rheological response beyond a critical stress and develop pronounced inhomogeneities or 'shear bands' even in apparently simple shearing deformations such as start-up of steady shear flow or large amplitude step strains. To capture the onset and evolution of these shear-banding transitions it is necessary to develop and solve appropriate constitutive models that couple the spatial and temporal evolution of both microstructural information (such as the number density and conformation of active species) as well as the resulting macroscopic stress resulting from the deformation of the microstructure.

In this work we are specially interested in the viscoelastic systems formed by wormlike micellar surfactants in a Newtonian solvent. In solution these "worms" can entangle, like polymers, and thus add elasticity to the fluid flow, but in addition they introduce an extra relaxation mechanism into the system due to their ability to break and reform continuously. These solutions have been the center of numerous theoretical and experimental studies as discussed in [1-4]. Applications of wormlike micellar solutions can be found in diverse industries: in the cosmetics industry they are used as gelation and shear thinning agents in products like shampoos and body gels; in the petroleum industry they are used in fracturing operations and enhanced oil recovery.

Of particular interest is the fact that flows of wormlike micellar solutions can become spatially inhomogeneous under simple loading conditions. Experiments have shown that under certain conditions of concentration and temperature, these solutions exhibit local shear banding events, leading to the formation of domains with distinct localized shear rates. In particular, in steady state shear flow experiments using a circular Couette geometry there is a range of apparent shear rates, $\dot{\gamma}_{1}<\dot{\gamma}<\dot{\gamma}_{2}$, for which the steady state velocity profile across the gap shows a greatly-enhanced velocity gradient towards the inner or moving cylinder and a much lower velocity gradient towards the outer or fixed cylinder [5-11]. The kinematics of this shear banding behavior has been studied experimentally by a number of groups using a range of localized probes such as flow induced birefringence [5,6], particle image velocimetry [6-8], light scattering and small angle neutron scattering [9], ultrasound [12], and nuclear magnetic resonance $[10,11]$. For such systems the corresponding steady state flow curves are measured in a rheometer.

In the steady state flow curve, for shear rates less than $\dot{\gamma}_{1}$, or larger than $\dot{\gamma}_{2}$, the variation in the velocity profile is almost linear across the gap as expected for a viscometric flow of a Newtonian fluid for which the velocity profile is given 
exactly by

$$
v(r)=v_{i} \frac{r_{i}}{r}\left[\frac{r_{o}^{2}-r^{2}}{r_{o}^{2}-r_{i}^{2}}\right]=\left(\frac{v_{i} r_{i} r_{o}^{2}}{r_{o}^{2}-r_{i}^{2}}\right) \frac{1}{r}-\left(\frac{v_{i} r_{i}}{r_{o}^{2}-r_{i}^{2}}\right) r
$$

where $v_{i}$ is the velocity of the inner cylinder, $v_{o}$ the velocity of the outer cylinder is zero, and $r_{i}, r_{o}$ are the inner and outer radius respectively. If $y \equiv \frac{r-r_{i}}{r_{o}-r_{i}}$ is the local distance across the gap, then for $p=\left(r_{o}-r_{i}\right) / r_{i}<<1$, the equation above can be linearized as

$$
v(r)=v_{i}(1-y)(1+O(p)) \approx v_{i}(1-y)
$$

in which $p$ is the curvature parameter. For shear rates in the intermediate regime, $\dot{\gamma}_{1}<\dot{\gamma}<\dot{\gamma}_{2}$, the velocity profile exhibits banded like variations which are substantially larger than $O(p)$. The steady state flow curve measured in a rheometer for many micellar solutions exhibits a plateau. Measurements with monodisperse entangled ploymer solutions also show a pronounced plateau [13]. It has been pointed out by Porte et al. [14] and Berret [15] that such a flow curve could result from an underlying non-monotonic shear stress versus shear rate curve such as the one shown in Figure 1.

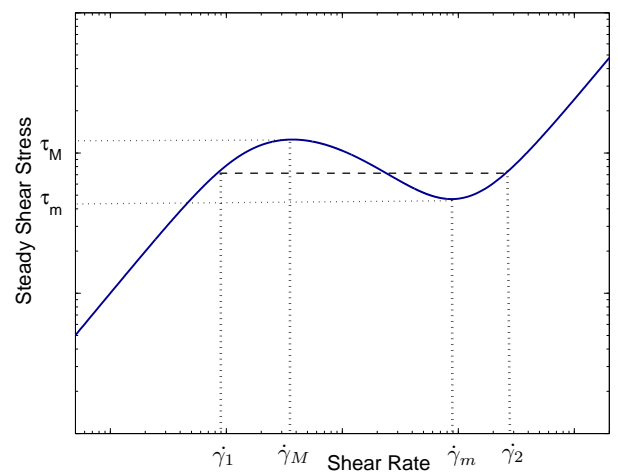

Figure 1: Steady state shear stress versus shear rate in viscometric flow. The stress region $\tau_{m}<\tau<\tau_{M}$ is a multi-valued section of the viscometric flow curve, shown here with the solid line. Experiments show that in this region shear banding occurs causing the formation of a stress plateau similar to the dashed curve.

Homogeneous solutions to the underlying constitutive equation in the region of the negative slope of the flow curve can be shown to be unstable [16]. Thus for shear rates/stresses in this region, the system is expected to select a stable composite solution which consists of two or more domains with shear rates on the rising portion of the curve that exist at the same shear stress values, as dictated by the momentum equation, in a such a way as to satisfy the external boundary conditions. This results in 'shear banding', or the development of multiple localized bands with different shear rates. In this case, since the actual 
flow is inhomogeneous, there is a marked difference between the apparent value of any variable observed in a rheometer and its local value. For example, the apparent shear rate $\dot{\gamma}_{a p p}$ can be defined, as in [17],

$$
\dot{\gamma}_{a p p}=\frac{r_{i}}{r_{o}-r_{i}} \int_{r_{o}}^{r_{i}} \frac{\dot{\gamma}(r)}{r} d r,
$$

where the expression on the right hand side comes from the proper curvilinear definition of the local shear rate in circular Couette flow,

$$
\dot{\gamma}(r)=r \frac{\partial}{\partial r}\left(\frac{v(r)}{r}\right) .
$$

Recent particle image velocity (PIV) measurements have shown that similar band-like transitions with strongly inhomogeneous local velocity gradients can also develop and evolve in highly monodisperse entangled polymer solutions undergoing both steady [13, 18] and transient shearing flows [19, 20].

Constitutive modeling of complex fluids that undergo shear banding deformations can be subdivided into two categories: (i) phenomenological models that describe global features such as the plateau in the flow curve but provide no information on local kinematic features such as shear bands (see for example [21]) and (ii) more complex models that result in a non-monotonic flow curve under the assumptions of viscometric deformation [22]. For further details and discussion of these approaches, see for example the recent reviews of $[3,4]$. In the latter class of models it is necessary to solve the coupled differential equations for stress and conservation of momentum in order to determine the local velocity field and the resulting apparent flow curve. In the present paper we compare our results with those predicted by the Johnson-Segalman model [23], which has been extensively studied in inhomogeneous steady shear flows $[22,24-27]$. Although motivated by physical processes such as non-affine chain deformation, the Johnson-Segalman (JS) class of models cannot be connected directly to microstructural features of the flow such as the breaking and reforming processes important in shear-banding transitions of wormlike micellar solutions. These processes lead to experimental observations of variations in the alignment and the number density of wormlike micelles in space and time $[9,28]$. In addition, in step strain calculations, the shear stress and normal stress differences predicted by the Johnson-Segalman model can become nonphysical and lead to oscillatory functions of strain (see discussion below and Figure 2). Furthermore, the predictions of the Johnson-Segalman model do not obey the Lodge-Meissner relationship [29] and in elongational flows, the axial component of the stress predicted by the JS model grows without bound in time [29] leading to divergence in the extensional viscosity. Thus viscoelastic constitutive models which capture the coupled evolution in local microstructure and stress, and which can be related to physical scission/reforming processes need to be formulated and investigated.

In [30] a network model focusing on two interacting species was formulated to describe the rheological behavior of wormlike micellar solutions. In this model, 
referred to hereafter as the VCM model, the micelles were modeled as elastic segments comprised of Hookean springs connected to form an elastic network. The breaking and reforming dynamics of the chains provide a "simplified" version of the reaction kinetics proposed by Cates [31]. The VCM model considers only two species of chains, but the chain breakage rates are allowed to depend on both the local shear rate and stress. Shorter chains, of length $L / 2$ units, can join one another at their ends to form into one long chain of length $L$ units. Analogously, a long chain can break in its center to form two short chains. This discrete two species model is greatly simplified as compared to the continuous Cates theory in which chains break with equal probability at any point along their length, and in which chains of any length can join to form a longer chain. However, the simplified formulation is amenable to studying nonlinear inhomogeneous and time dependent deformations. In [30] this two species VCM model was analyzed in the linear viscoelastic regime as well as in steady shear flow, step strain deformation and uniaxial extensional flow, but always under the assumption of viscometric flow conditions. Representative values of the five parameters of the model were also determined by comparison with rheological measurements in a well-studied wormlike micellar solution. However, quantitative comparison between the model predictions and observations are limited to small/moderate Weissenberg numbers $(W i<1)$ because the flow curve measured in steady shearing experiments exhibits the familiar plateau behavior sketched in Figure 1 (the broken line) whereas if a homogeneous flow is assumed a priori, the VCM model predicts the non-monotonic curve shown by the solid line in Figure 1.

In this paper we study computationally the onset and temporal evolution of inhomogeneous states in steady and transient shearing flows. The analysis and computations are carried out in a circular Couette geometry in which the global variation in the state of stress across the gap can be varied by controlling the ratio $r_{i} / r_{o}$. Because of the complexity of the coupled differential equations governing the evolution in microstructure and the local stress in the VCM class of network models, we first consider two special limiting cases: The first case is referred to as the PEC model since it focuses on a single viscoelastic species in which the functional form of the breakage rate of the long chains is described by a non-linear function that leads to the Partially Extending Convective strand constitutive equation proposed by Larson [32]. The Larson-PEC model was first introduced as a generalized differential analogue of the Doi-Edwards reptation theory for entangled polymer melts [29]. The second limiting case, referred to here as $\mathrm{PEC}+\mathrm{M}$, is a model with two non-interacting viscoelastic species in which the first species (species ' $\mathrm{A}$ ', consisting of longer and more elastic chains) evolves in a form consistent with the Larson-PEC model and the second species (species 'B', consisting of shorter and less elastic chains) is described by the upper convected Maxwell model. Such a model may be expected to accurately capture the key features of the rheological response observed in recent experiments with solutions of monodisperse entangled chains (species 'A') in solutions of shorter chains (species 'B') [18, 19].

As we discussed earlier, an alternate single species model, the JohnsonSegalman model, has been studied in shear by Olmsted et al.in, for example, 
$[22,27]$. We also re-computed those results, both as a baseline for verification of our numerical methods and in order to consistently compare the predictions from that model with the predictions from the two new models studied in the present work. All the models considered in this paper predict shear banding behaviors that qualitatively agree with experimental observations in steady shear flow. However, as noted above, the Johnson-Segalman model can predict unphysical responses in both step strain and extensional flow experiments [29], in contrast to the PEC and PEC+M models. To demonstrate this, in Figure 2 we compare the damping function predicted by the PEC model and the Johnson-Segalman model in step strain. Although both models show strain softening at strains of magnitude $\gamma>1$, the PEC model shows a smooth monotonic strain softening consistent with equivalent experimental observations in micellar solutions [30] and entangled polymer solutions [29].

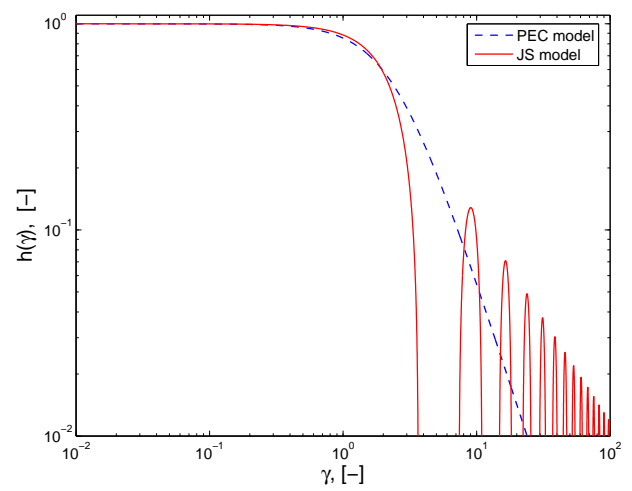

Figure 2: Viscometric calculations of the damping function from the PEC model with nonlinear parameter $\xi=0.52$ and the Johnson-Segalman model with nonlinear parameter $a=0.52$.

The structure of this paper is as follows, the formulation of the VCM model [30], which includes spatial variations as well as scission and reforming reactions of two interacting species is reviewed briefly in Section 2. In Section 3 the simplified PEC and PEC+M model forms are introduced, including the appropriate diffusion terms and additional Newtonian solvent contributions, and in Section 3.1 the governing partial differential equations are written out for time-dependent circular Couette flow. In Section 3.2 we present the boundary and initial conditions used in the numerical calculations. In Section 3.3-3.5, we give explicit solutions in shear and step strain for the PEC and PEC+M model assuming a homogeneous viscometric flow. A temporal stability analysis of the solutions along the flow curve and the effects of varying the different constitutive parameters appearing in the model on the stability of these solutions are also presented. Section 4 is a discussion of "apparent" gap-averaged (i.e. rheometrically-observable quantities) versus "local" variations of the stress. 
Finally, in Section 5, steady state as well as time-dependent computational results are presented for the PEC and PEC+M models in start-up of steady shear flow and in step strain. These are the first computations we are aware of that describe the evolution of shear bands following a sudden step strain using a viscoelastic constitutive model with an underlying non-monotonic viscometric flow curve. The computational predictions for the structure and evolution of the shear bands in start-up of steady shear flow capture many of the features observed in recent PIV measurements with entangled polymer solutions [18, 19] as well as observations of shear-banding in strong flows of wormlike micellar solutions.

\section{Governing Equations for the Two Species Scis- sion Network Model}

In the VCM model we consider chains of length $L$, labeled species $A$, and chains of length $L / 2$, labeled species $B$. Using the tools of kinetic theory, as in $[30,33-$ $35]$, one can derive the evolution equations for the number density probability distribution functions of species $A$ and $B, \Psi_{A}(\mathbf{r}, \mathbf{Q}, t)$ and $\Psi_{B}(\mathbf{r}, \mathbf{Q}, t)$ respectively, considering variations in space, configuration space, and time. The details can be found in [30]. Here $\mathbf{Q}$ is the connector vector between elastically-active nodes of the network. Integrating these equations over the configuration space, $d \mathbf{Q}$, and non-dimensionalizing as in [30], gives the equations for the number density of the two species defined as

$$
\begin{aligned}
& n_{A}=\int \Psi_{A} d \mathbf{Q}, \\
& n_{B}=\int \Psi_{B} d \mathbf{Q} .
\end{aligned}
$$

Similarly, multiplying by QQ and then integrating over configuration space gives the equations for the non-dimensional stress contributions from species $A$ and $B$ as

$$
\begin{aligned}
& \mathbf{A}=\int \mathbf{Q Q} \Psi_{A} d \mathbf{Q}=\{\mathbf{Q Q}\}_{A}, \\
& \mathbf{B}=\int \mathbf{Q Q} \Psi_{B} d \mathbf{Q}=\{\mathbf{Q Q}\}_{B} .
\end{aligned}
$$

Combining these expressions with the Smoluchowski equation gives the governing equations for the dimensionless number densities:

$$
\begin{aligned}
\mu \frac{D n_{A}}{D t} & =2 \delta_{A} \nabla^{2} n_{A}-\delta_{A} \nabla \nabla: \mathbf{A}+\frac{c_{B}}{2} n_{B}^{2}-c_{A} n_{A} \\
\mu \frac{D n_{B}}{D t} & =2 \delta_{B} \nabla^{2} n_{B}-2 \delta_{B} \nabla \nabla: \mathbf{B}-c_{B} n_{B}^{2}+2 c_{A} n_{A}
\end{aligned}
$$


and for the dimensionless stress contributions:

$$
\begin{aligned}
\mu \mathbf{A}_{(1)}+\mathbf{A}-n_{A} \mathbf{I}-\delta_{A} \nabla^{2} \mathbf{A} & =c_{B} n_{B} \mathbf{B}-c_{A} \mathbf{A} \\
\epsilon \mu \mathbf{B}_{(1)}+\mathbf{B}-\frac{1}{2} n_{B} \mathbf{I}-\epsilon \delta_{B} \nabla^{2} \mathbf{B} & =\epsilon\left(-2 c_{B} n_{B} \mathbf{B}+2 c_{A} \mathbf{A}\right)
\end{aligned}
$$

where $(\cdot)_{(1)}$ indicates the upper convected derivative

$$
(\cdot)_{(1)}=\frac{\partial(\cdot)}{\partial t}+\mathbf{v} \cdot \nabla(\cdot)-\left((\nabla \mathbf{v})^{\top} \cdot(\cdot)+(\cdot) \cdot \nabla \mathbf{v}\right) .
$$

The nondimensional total micellar stress is given by

$$
\boldsymbol{\sigma}=\mathbf{A}+2 \mathbf{B}
$$

The parameter $\mu=\lambda_{A} / \lambda_{\text {eff }}$ is the ratio of the relaxation time of species $A$ in the absence of scission and reforming (reptation time) to the effective relaxation time of the solution when scission and reforming dynamics are included. The parameter $\epsilon=\lambda_{B} / \lambda_{A}$ is the ratio of the relaxation time of the shorter species $B$ to that of the longer species $A$. The breaking and reforming rates, $c_{A}$ and $c_{B}$ remain to be specified. In [30] we consider

$$
\begin{aligned}
& c_{A}=c_{A \text { eq }}+\frac{1}{3} \xi\left(\dot{\gamma}: \frac{\mathbf{A}}{n_{A}}\right) \\
& c_{B}=c_{B \text { eq }}=\text { constant }
\end{aligned}
$$

where $\dot{\gamma}$ is the shear rate, $c_{A \text { eq }}$ and $c_{B \text { eq }}$ are the equilibrium breaking and reforming rates and $\xi$ is a parameter. The non-dimensional diffusion constants are defined as $\delta_{A}=\lambda_{A} D_{A} / h^{2}$ and $\delta_{B}=\delta_{A} / 2$, where $h$ is the dimensional gap width and $D_{A}$ is the diffusivity of species A (see [30]). These parameters are important in the analysis of inhomogeneous flows and lead to the development of boundary/internal layers in such flows [34]. They also result in smoothing of sharp discontinuities that can otherwise arise between neighboring shear-bands. These diffusion terms, introduced by Bhave et al. in [34] require specification of boundary conditions on the stress at the walls. In [34] Dirichlet conditions were imposed resulting in boundary layers at the walls. Alternate Neumann boundary conditions are considered in [24]. For further discussion on the variations in the rheological predications as the model parameters are varied see [30].

The constitutive equations (8) for the total viscoelastic stress and the evolution equations for the number densities of the two species (7) are coupled

with conservation equations for mass and momentum, which can be written in dimensionless form as:

- Conservation of Mass

$$
\boldsymbol{\nabla} \cdot \mathbf{v}=0
$$

- Conservation of Momentum

$$
E^{-1} \frac{\partial \mathbf{v}}{\partial t}=-\nabla \cdot\left(p \mathbf{I}-\beta \dot{\gamma}+\boldsymbol{\tau}_{\boldsymbol{p}}\right)
$$


where $E=\lambda_{\mathrm{eff}} \eta_{0} / \rho h^{2}$ is the elasticity number which measures the relative importance of unsteady elastic and inertial effects in the flow [30]. The solvent viscosity ratio is given by $\beta=\eta_{s} / \eta_{0}$ and is typically very small for micellar fluids or concentrated polymer solutions. The total polymeric contribution to the stress is comprised of the contributions from species A and B plus an additional isotropic contribution from each species:

$$
\boldsymbol{\tau}_{\boldsymbol{p}}=-\mathbf{A}-2 \mathbf{B}+\left(n_{A}+n_{B}\right) \mathbf{I}
$$

\section{The PEC and PEC+M Models}

The first nonlinear network model examined in this paper is a single species model that is identical in homogeneous shear flow to the differential constitutive equation proposed by Larson [32] for entangled polymer melts undergoing reptation. The partial retraction of the chains inside the convecting and deforming tubes is described by the Partially Extended Convected derivative and is referred to henceforth as the PEC model. The second model, denoted for brevity as PEC $+\mathrm{M}$ considers two non-interacting viscoelastic species, in which the first species is modeled by the Larson-PEC model and the second species by the upper convected Maxwell model. Analyses of these two models in inhomogeneous flows, either with or without diffusion, have not been considered previously, and are a first step to considering our full scission/reforming network model for entangled wormlike micellar fluids. These two models are also relevant to understanding the shear banding observed in strong shear flows of monodisperse polymer melts $[18,19]$. The rheological predictions of the two models in homogeneous flow are summarized in Figure 3. In the PEC model the presence of a viscous Newtonian solvent contribution is crucial to assuring that there is an upturn in the steady state viscometric flow curve at high shear rates. In the two mode PEC+M model, the shorter and less elastic Maxwell species takes the place of this solvent, and thus physically-realistic values for the additional Newtonian solvent can be used, as shown in Figure 3(b). Note that for a wormlike micellar solution with a zero shear rate viscosity $\eta_{0}=14.5 \mathrm{~Pa}$-s, the non-dimensional solvent viscosity $\beta=\eta_{s} / \eta_{0}=6.78 \times 10^{-5}$ corresponds to a solvent viscosity $\eta_{s}=0.001 \mathrm{~Pa}$-s which is the viscosity of water. In principle, the PEC $+\mathrm{M}$ model is well-posed and can also be studied in the limit $\beta \rightarrow 0$, however the presence of a small but finite solvent viscosity is important in the numerical algorithm employed for solving inhomogeneous flow profiles; we thus retain this term in our analysis below. Finally, the inserts in Figure 3 show the dimensionless first normal stress difference $N_{1}$ as a function of shear rate. In the single mode PEC model, $N_{1}$ approaches a plateau at high shear rates due to increasing chain disentanglement and retraction. By contrast, in the two species $\mathrm{PEC}+\mathrm{M}$ model the second viscoelastic mode (describing the shorter disentangled chains) contributes additional elasticity to the fluid response at high shear rates, which is consistent with experimental findings [36]. 


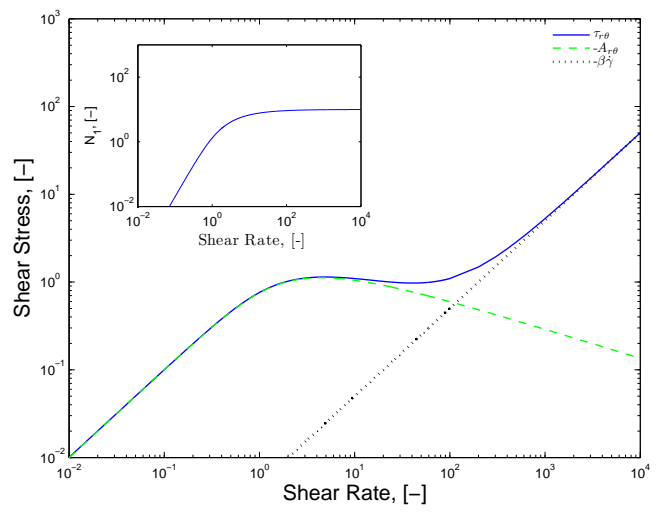

(a)

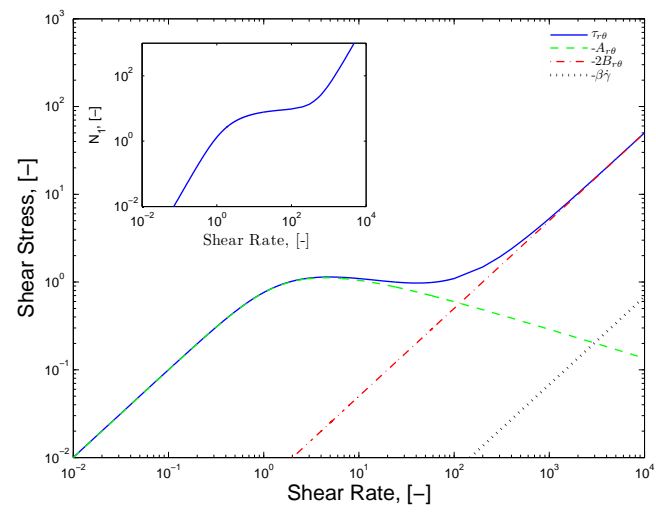

(b)

Figure 3: (a) Viscometric steady state shear stress for the PEC model with $\xi=0.3$ and $\beta=0.005$; in this model $\tau_{r \theta}=-A_{r \theta}-\beta \dot{\gamma}$. (b) Viscometric steady state shear stress for the PEC $+\mathrm{M}$ model with $\xi=0.3, \epsilon n_{B}=0.005$, and $\beta=6.78 \times 10^{-5}$; in this model $\tau_{r \theta}=-A_{r \theta}-2 B_{r \theta}-\beta \dot{\gamma}$. Inserts: First normal stress differences.

\section{- PEC Model with Conformation-Dependent Diffusion}

The PEC form of the VCM model, with the addition of the diffusion terms that arise in the formulation when gradients of local conformations are considered [29], is

$$
\mathbf{A}_{(1)}+\mathbf{A}-\mathbf{I}-\delta_{A} \nabla^{2} \mathbf{A}=-\frac{\xi}{3}(\dot{\gamma}: \mathbf{A}) \mathbf{A}
$$

where the micellar stress is given by $\boldsymbol{\sigma}=\mathbf{A}$ (Equation (10) with $\mathbf{B}=\mathbf{0}$ ).

This model can be obtained as a limiting case of the VCM model, when $n_{A}=1, n_{B}=0$ and $\epsilon=c_{A \text { eq }}=c_{B \text { eq }}=0$, that is, there is no equilibrium breakage or reforming of the longer species $A$ chains. In this limit $\mu=1$ (or $\lambda_{\text {eff }}=\lambda_{A}$ ) so that the effective relaxation time of the mixture, $\lambda_{\text {eff }}$, is equal to the reptation time, $\lambda_{A}$, of species $\mathrm{A}$ and there is no re-scaling of the effective relaxation time of the elastic network from the breakage of chains. The PEC model without diffusion terms was formulated by Larson as a differential form of the Doi-Edwards model for entangled polymer melts undergoing reptation [29]. The polymer chains, in this case, are allowed partial, versus full, retraction within their tubes. This process is governed by the non-linear term on the right hand side of Equation (15) and controlled by the magnitude of parameter $\xi$. In the PEC model such a term represents partial tube loss following chain retraction as opposed to micelle breakage as in the VCM model. In the latter case, an evolution equation for number density is also required for mathematical consistency but otherwise the resulting constitutive formulation is identical. Hence the PEC model should be a good differential model for fast flows of monodisperse and entangled polymeric solutions where there is no chain breakage $[18,19]$. 


\section{- The PEC+M Model with Conformation-Dependent Diffusion}

The PEC $+\mathrm{M}$ model is introduced as a precursor to consideration of the full VCM interacting species model. The constitutive equations for the contributions to the total stress from the two active species in the elastic network are

$$
\begin{aligned}
\mathbf{A}_{(1)}+\mathbf{A}-\mathbf{I}-\delta_{A} \nabla^{2} \mathbf{A} & =-\frac{\xi}{3}(\dot{\gamma}: \mathbf{A}) \mathbf{A} \\
\epsilon \mathbf{B}_{(1)}+\mathbf{B}-\frac{n_{B}}{2} \mathbf{I}-\epsilon \delta_{B} \nabla^{2} \mathbf{B} & =\mathbf{0},
\end{aligned}
$$

where the total viscoelastic stress is $\boldsymbol{\sigma}=\mathbf{A}+2 \mathbf{B}$. Here we expect $\delta_{A}, \delta_{B}$, and $\epsilon=\lambda_{B} / \lambda_{A}$ to be much smaller than one. These three parameters are defined as in Section 2. As in the single species case, $\mu=1$ since this model considers no breaking or reforming of the chains.

There is no specific limiting procedure on the VCM model that will generate the $\mathrm{PEC}+\mathrm{M}$ model since conservation of species in the $\mathrm{VCM}$ requires a term of the form $(2 \xi / 3)(\dot{\gamma}: \mathbf{A}) \mathbf{A}$ in the right hand side of Equation (16b). However, there are a number of similarities between this model and the VCM model that are worth investigating. The model (16) can be understood as combining the stresses from two non-interacting viscoelastic species, the longer chains 'A' under reptative like motion (with reptation time $\lambda_{A}$ ) and the shorter unentangled chains ' $\mathrm{B}$ ' (with relaxation time $\lambda_{B}<<\lambda_{A}$ ) which give rise to Rouse-like viscoelasticity. This formulation should be a good model to describe the experiments in $[18,19]$ which consider solutions of long polybutadiene chains $\left(\mathrm{MW} \sim 10^{6} \mathrm{~g} / \mathrm{mol}, \mathrm{c} \sim 10 \%\right)$ in a sea of short chains $\left(\mathrm{MW} \sim 10^{3} \mathrm{~g} / \mathrm{mol}\right)$.

\subsection{Shear Flow Equations in Circular Couette Geometry}

In the circular Couette geometry we assume a unidirectional shearing flow of the form

$$
\mathbf{v}=(0, v(r), 0)
$$

so that

$$
\begin{aligned}
(\mathbf{v} \cdot \nabla)(\cdot) & =0 \\
\dot{\gamma} & =\dot{\gamma} \boldsymbol{\delta}_{r} \boldsymbol{\delta}_{\theta}+\dot{\gamma} \boldsymbol{\delta}_{\theta} \boldsymbol{\delta}_{r} \\
\dot{\gamma}(r) & =r \frac{\partial}{\partial r}\left(\frac{v}{r}\right) \\
\dot{\gamma}: \mathbf{A} & =2 \dot{\gamma} A_{r \theta} \\
\nabla \nabla:(\cdot) & =\frac{1}{r} \frac{\partial^{2}}{\partial r^{2}}\left(r(\cdot)_{r r}\right)-\frac{1}{r} \frac{\partial}{\partial r}(\cdot)_{\theta \theta},
\end{aligned}
$$

where $\dot{\gamma}(r)$ is the local shear rate. With these assumptions conservation of mass is automatically satisfied, and the two relevant conservation of momentum equations are:

$$
E^{-1} \frac{\partial v}{\partial t}=\frac{1}{r^{2}} \frac{\partial}{\partial r}\left(r^{2}\left(-\tau_{p r \theta}+\beta \dot{\gamma}\right)\right)
$$


and

$$
E^{-1} \frac{v^{2}}{r}=\left(\frac{1}{r} \frac{\partial}{\partial r}\left(r \tau_{p r r}\right)-\frac{\tau_{p \theta \theta}}{r}\right)+\frac{\partial p}{\partial r}
$$

where

$$
\begin{aligned}
& \tau_{p r r}=-A_{r r}-2 B_{r r}+\left(1+n_{B}\right) \\
& \tau_{p r \theta}=-A_{r \theta}-2 B_{r \theta} \\
& \tau_{p \theta \theta}=-A_{\theta \theta}-2 B_{\theta \theta}+\left(1+n_{B}\right) .
\end{aligned}
$$

For the PEC model $n_{B}=0$ and $\mathbf{B}=0$. As pointed out at the beginning of this section, the values of $\beta$ are of different orders of magnitude for the PEC and the PEC $+\mathrm{M}$ models. In the PEC model the stress contributions at large shear rates come solely from the solvent viscosity. To fit the measured upturn in the shear stress in wormlike micellar systems, for this range of shear rates, an unphysically large value of the solvent viscosity is assumed $\left(\beta=O\left(10^{-3}\right)\right)$. On the other hand, in the $\mathrm{PEC}+\mathrm{M}$ model such contributions to the stress are given by the viscoelastic species $B$ and the solvent viscosity takes a more realistic value so that $\beta=\eta_{s} / \eta_{0}=O\left(10^{-5}\right)$.

\section{- PEC Model with Conformation-Dependent Diffusion: Circular Couette Equations}

From Equation (15) and Equations (17)-(21), we obtain the governing equations for the stress components in the cylindrical Couette geometry as follows

$$
\begin{aligned}
\frac{\partial A_{r r}}{\partial t}+A_{r r}-1-\delta_{A}\left[\frac{1}{r} \frac{\partial}{\partial r}\left(r \frac{\partial}{\partial r} A_{r r}\right)\right. & \left.-\frac{2}{r^{2}}\left(A_{r r}-A_{\theta \theta}\right)\right] \\
& =-\frac{2}{3} \xi \dot{\gamma} A_{r \theta} A_{r r} \\
\frac{\partial A_{r \theta}}{\partial t}-\dot{\gamma} A_{r r}+A_{r \theta}-\delta_{A}\left[\frac{1}{r} \frac{\partial}{\partial r}\left(r \frac{\partial}{\partial r} A_{r \theta}\right)\right. & \left.-\frac{4}{r^{2}} A_{r \theta}\right] \\
& =-\frac{2}{3} \xi \dot{\gamma} A_{r \theta} A_{r \theta} \\
\frac{\partial A_{\theta \theta}}{\partial t}-2 \dot{\gamma} A_{r \theta}+A_{\theta \theta}-1-\delta_{A}\left[\frac{1}{r} \frac{\partial}{\partial r}\left(r \frac{\partial}{\partial r} A_{\theta \theta}\right)\right. & \left.+\frac{2}{r^{2}}\left(A_{r r}-A_{\theta \theta}\right)\right] \\
& =-\frac{2}{3} \xi \dot{\gamma} A_{r \theta} A_{\theta \theta} \\
\frac{\partial A_{z z}}{\partial t}+A_{z z}-1-\delta_{A}\left[\frac{1}{r} \frac{\partial}{\partial r}\left(r \frac{\partial}{\partial r} A_{z z}\right)\right] & \\
& =-\frac{2}{3} \xi \dot{\gamma} A_{r \theta} A_{z z} .
\end{aligned}
$$




\section{- PEC+M Model with Conformation-Dependent Diffusion: Circular Couette Equations}

In the PEC $+\mathrm{M}$ model, the contributions of the longer chain species are modeled by the PEC model and the stress components of species $A$ are again given by Equations (24). These equations are coupled, through the momentum equations, with the equations for the contributions to the stress from the shorter chain species $B$ obtained from Equation (16b),

$$
\begin{aligned}
& \epsilon \frac{\partial B_{r r}}{\partial t}+B_{r r}-\frac{n_{B}}{2}-\epsilon \delta_{B}\left[\frac{1}{r} \frac{\partial}{\partial r}\left(r \frac{\partial}{\partial r} B_{r r}\right)-\frac{2}{r^{2}}\left(B_{r r}-B_{\theta \theta}\right)\right] \\
& =0 \\
& \epsilon \frac{\partial B_{r \theta}}{\partial t}-\epsilon \dot{\gamma} B_{r r}+B_{r \theta}-\epsilon \delta_{B}\left[\frac{1}{r} \frac{\partial}{\partial r}\left(r \frac{\partial}{\partial r} B_{r \theta}\right)-\frac{4}{r^{2}} B_{r \theta}\right] \\
& =0 \\
& \epsilon \frac{\partial B_{\theta \theta}}{\partial t}-2 \epsilon \dot{\gamma} B_{r \theta}+B_{\theta \theta}-\frac{n_{B}}{2}-\epsilon \delta_{B}\left[\frac{1}{r} \frac{\partial}{\partial r}\left(r \frac{\partial}{\partial r} B_{\theta \theta}\right)+\frac{2}{r^{2}}\left(B_{r r}-B_{\theta \theta}\right)\right] \\
& =0 \\
& \epsilon \frac{\partial B_{z z}}{\partial t}+B_{z z}-\frac{n_{B}}{2}-\epsilon \delta_{B}\left[\frac{1}{r} \frac{\partial}{\partial r}\left(r \frac{\partial}{\partial r} B_{z z}\right)\right] \\
& =0 \text {. }
\end{aligned}
$$

We seek to describe systems in which the shorter species $B$ follow unentangled Rouse-like dynamics so that $\lambda_{B} \sim(L / 2)^{2}$, and the A species relaxes through reptation so that $\lambda_{A} \sim L^{3} / L_{E}$, where $L_{E}$ is the entanglement length [30], and hence $\epsilon=\lambda_{B} / \lambda_{A} \sim L_{E} / 4 L<<1$. In the limit $\epsilon \rightarrow 0$, species $B$ behaves like a Newtonian fluid with a purely Newtonian contribution to the stress which in dimensional terms is $\eta_{B}=n_{B} k T \lambda_{B}[30]$.

\subsection{Boundary and Initial Conditions}

In this paper we consider two types of deformations: start up of steady simple shear flow and stress relaxation after a step shear strain. In the first case we impose the apparent shear rate or velocity across the gap (see equation (3)), in the second case we control the apparent strain across the gap by imposing a displacement at the inner wall.

- Imposed Shear at the Walls

For controlled shear rate experiments, ideally the boundary conditions are:

$$
\begin{aligned}
v_{i}\left(t=0^{-}\right)=0, \quad v_{i}\left(t=0^{+}\right)=v_{i} & =D e \\
v_{o} & =0 .
\end{aligned}
$$


Here $D e=\lambda_{A} V^{\prime} / h$, where $V^{\prime}$ is the dimensional velocity at the inner cylinder [30]. Note that we do not consider the possible effects of slip at the wall, although this is observed in some experiments [19]. We assume the walls are suitably roughened to eliminate any slip effects. Because it is impossible in practice to achieve an instantaneous step jump in the wall velocity, we consider two different possible approaches to imposing shear at the inner cylinder; the first a "quick" ramp, or jump-up, in shear rate, the second a slow ramp in shear rate. Similar cases are considered by Olmsted et al. [22]. For both conditions the velocity at the inner wall, for $t \geq 0$, is modeled by

$$
v_{i}(t)=D e \tanh (a t),
$$

where time is scaled with $\lambda_{\mathrm{A}}$. This velocity corresponds to an eventual nondimensional apparent shear rate across the gap at steady state of $\dot{\gamma}_{a p p}=D e$. In the first, 'jump-up' case, we select the dimensionless coefficient $a$ such that the ramp is complete well before the relaxation time of species $A$. We chose $a=2$ for which the time to half-height for the tanh function is roughly a quarter of one relaxation time. In the second, 'ramp up' case we use a ramp that is not complete until after several relaxation times. We chose $a=0.1$, hence it takes roughly 5 to 6 relaxation times to get to half-height. The effect that these two functions have on the predictions of the models, as well as the effect of even faster jumps, will be studied in Section 5 .

If the diffusion parameters $\delta_{A}, \delta_{B}$ are not zero, boundary conditions on the stress must be imposed at the walls. There has been some discussion in the literature as to the appropriate boundary conditions to impose [37]. In the present computations, we use no flux of conformation as used by [22, 24, 34, 38]. The Neumann boundary condition for the stress at the walls are then:

$$
\begin{aligned}
& \left.\frac{\partial \mathbf{A}}{\partial r}\right|_{i}=\left.\frac{\partial \mathbf{A}}{\partial r}\right|_{o}=0, \\
& \left.\frac{\partial \mathbf{B}}{\partial r}\right|_{i}=\left.\frac{\partial \mathbf{B}}{\partial r}\right|_{o}=0 .
\end{aligned}
$$

Some analysis of imposing Dirichlet as opposed to Neumann boundary conditions on the stress can be found in [39].

\section{- Imposed Step Strain at the Wall}

In step strain experiments, the response of the fluid to an instantaneous imposed strain is considered. However in practice because of the finite moment of inertia for any rheometric fixture, the displacement of the inner cylinder can not be completed in zero time, and hence in our computations we use a ramp in strain thus specifying the inner wall displacement as

$$
d_{i}(t)=\gamma_{0}(1-(1+b t) \exp (-b t)) .
$$

The displacement of the outer cylinder is fixed, so that

$$
d_{o}(t)=0 .
$$


Equation (29) corresponds to an applied velocity at the inner cylinder of

$$
v_{i}(t)=\gamma_{0} b^{2} t \exp (-b t) .
$$

Here we explore the effect of different values of the parameter $b$, where $b$ is a dimensionless measure of the motor response rate. The velocity of the cylindrical bob starts at zero, rises rapidly and then decays to a final rest state after the desired angular strain displacement is attained. Calculations of ideal steps in strain are given as limiting cases when $b \rightarrow \infty$.

\section{- Initial Conditions}

All computations in this paper are carried out in time to capture the history dependence of the viscoelastic stress fields. Conditions at $t=0^{-}$for both ramp up and jump up tests are that the network is at resting equilibrium, so that the stress satisfies

$$
\begin{aligned}
& A_{r \theta}(r, 0)=B_{r \theta}(r, 0)=0, \\
& A_{r r}(r, 0)=A_{\theta \theta}(r, 0)=1, \\
& B_{r r}(r, 0)=B_{\theta \theta}(r, 0)=\frac{n_{B}}{2} .
\end{aligned}
$$

\section{3 $\mathrm{PEC} / \mathrm{PEC}+\mathrm{M}$ in Viscometric Shear Flow}

In steady state shearing flow, the solution to the PEC model can be found explicitly as a function of the shear rate if spatial dependence in the shear rate is ignored (viscometric flow). From Equations (24) setting $\dot{\gamma}$ to be a constant, the normal components of the conformation as a function of the shear stress and shear rate are [32]:

$$
A_{r r}=\frac{1}{1+\frac{2}{3} \xi \dot{\gamma} A_{r \theta}}, \quad A_{\theta \theta}=\frac{1+2 \dot{\gamma} A_{r \theta}}{1+\frac{2}{3} \xi \dot{\gamma} A_{r \theta}}
$$

so that

$$
N_{1}=A_{\theta \theta}-A_{r r}=\frac{6 \dot{\gamma} A_{r \theta}}{3+2 \xi \dot{\gamma} A_{r \theta}},
$$

where $N_{1}$ is the dimensionless first normal stress difference scaled with $n_{A} k T$, and the shear rate is scaled with $\lambda_{A}$. Substituting these expressions into Equation (24b) gives

$$
\frac{4}{9} \xi^{2} \dot{\gamma}^{2} A_{r \theta}^{3}+\frac{4}{3} \xi \dot{\gamma} A_{r \theta}^{2}+A_{r \theta}-\dot{\gamma}=0 .
$$

The only real solution to this cubic equation is

$$
A_{r \theta}=\frac{1}{2 \xi \dot{\gamma}} \frac{(J-1)^{2}}{J}
$$

where

$$
J=\left(1+9 \xi \dot{\gamma}^{2}+3 \xi \dot{\gamma} J_{0}\right)^{1 / 3} \quad \text { and } \quad J_{0}=\sqrt{\frac{2+9 \xi \dot{\gamma}^{2}}{\xi}}
$$


The curve described by Equation (36) is non-monotonic, with a maximum at

$$
\dot{\gamma}_{\max }=\sqrt{\frac{6}{\xi}} \quad \text { and } \quad A_{r \theta \max }=\frac{1}{4} \sqrt{\frac{6}{\xi}} .
$$

Furthermore, asymptotically for $\dot{\gamma}<<1$, we obtain the expected form

$$
A_{r \theta} \sim \dot{\gamma} \quad \text { and } \quad N_{1} \sim 2 \dot{\gamma}^{2},
$$

whereas for $\dot{\gamma}>>1$,

$$
A_{r \theta} \sim\left(\frac{3}{2 \xi}\right)^{2 / 3} \dot{\gamma}^{-1 / 3}-\frac{1}{3} \dot{\gamma}^{-1} \quad \text { and } \quad N_{1} \sim \frac{3}{\xi}-2\left(\frac{3}{2 \xi}\right)^{4 / 3} \dot{\gamma}^{-2 / 3}
$$

Therefore, when the solvent viscosity $\beta \dot{\gamma}$ is added to the micellar contribution to the shear stress, $A_{r \theta}$, with the right choices of $\beta$ and $\xi$, the total shear stress first increases with shear rate, then decreases, as $A_{r \theta}$ decreases, and finally increases again as $A_{r \theta}$ decays away and the solvent shear stress $\beta \dot{\gamma}$ dominates (see Figure 3(a)). Similarly, the first normal stress difference first increases quadratically in shear rate, then levels off to a constant value $3 / \xi$ (see the insert in Figure 3(a)).

For the PEC $+\mathrm{M}$ model the micellar contribution to the stress is $\boldsymbol{\sigma}=\mathbf{A}+2 \mathbf{B}$, where we expect $\epsilon=\lambda_{B} / \lambda_{A}<<1$. When $\dot{\gamma}$ is small, the total stresses come primarily from species A. When $\epsilon \dot{\gamma}>1$ (so $\dot{\gamma}>>1$ ),

$$
\begin{aligned}
\sigma_{r \theta} & \sim 2 B_{r \theta}=\epsilon n_{B} \dot{\gamma} \\
N_{1} & \sim 2\left(B_{\theta \theta}-B_{r r}\right)=(\epsilon \dot{\gamma})^{2} n_{B} .
\end{aligned}
$$

Thus, in the limit of $\dot{\gamma}>>1$, the contribution from species B dominates the total viscoelastic response of the network (see Figure 3(b)). The stress contribution from the solvent is negligible due to the small value of $\beta$. Note that in the PEC model $N_{1}$ approaches a constant value at large shear rates, since the Newtonian solvent provides no contribution to the first normal stress difference. Whereas for the PEC $+\mathrm{M}$ the curve for $N_{1}$ has an up-turn when $\epsilon \dot{\gamma}>1$ (so $\dot{\gamma}>>1$ ), this comes from the contribution from the viscoelastic species B. In contrast to the predictions of many empirical models, measurements in entangled micellar fluids do not typically show a plateau in $N_{1}[3]$ and the PEC+M model is thus expected to be a better description of the rheology of such fluids at high shear rates.

\subsection{Stability Analysis of the PEC Model}

To examine the stability of Equations (24) with $\delta_{A}=0$, we follow the results obtained by Yerushalmi et al. [16] and evaluate the dependence of the stability of the PEC model on its parameters, namely $\beta, \xi$. With the solutions as given 
in (33) and (36) as the base state, we perturb $\mathbf{A}$ and $\dot{\gamma}$ as

$$
\begin{aligned}
A_{r \theta} & =A_{r \theta}^{0}+A_{r \theta}^{1} e^{-\nu t}, \\
A_{r r} & =A_{r r}^{0}+A_{r r}^{1} e^{-\nu t}, \\
A_{\theta \theta} & =A_{\theta \theta}^{0}+A_{\theta \theta}^{1} e^{-\nu t}, \\
\dot{\gamma} & =\dot{\gamma}^{0}+\dot{\gamma}^{1} e^{-\nu t} .
\end{aligned}
$$

Figure 4 shows the base state steady flow curve, shear stress as a function of shear rate, for several values of $\xi$ and $\beta$. Also drawn in this figures is the curve of the smallest eigenvalue, $\nu$, of the perturbed system. The system is unstable $(\nu<0)$ in precisely those places where the flow curve has a negative slope. In general, the maximum and minimum points in the curve of shear stress versus shear rate, or equivalently, the bifurcation points of the curve as given by the change in sign of the real part of $\nu$, are given by

$$
\left.\dot{\gamma}\right|_{\max }=f(\beta) \sqrt{\frac{3}{2 \xi}},\left.\quad \dot{\gamma}\right|_{\min }=g(\beta) \sqrt{\frac{3}{2 \xi}} .
$$

Values of $f(\beta)$ and $g(\beta)$ for the values of $\beta$ used in this study are given in the caption of Figure 4. In [40], a general equation that gives the dependence of $\nu$ on $\beta$, $\xi$, and $\dot{\gamma}$ is given. The results in Figure 4(a) show that increasing the parameter $\xi$ results in a shift of the unstable region to smaller shear rates along the shear rate axis, in agreement with the predictions above for the location of the local maximum and local minimum of the curve. On the other hand, as seen in Figure 4(b), increasing $\beta$ lifts the eigenvalue curve so that for $\beta=0.016$ there is no longer a negative eigenvalue, while at the same time the flow curve has become monotonic.

\section{5 $\mathrm{PEC} / \mathrm{PEC}+\mathrm{M}$ in Viscometric Step Strain}

In a viscometric step strain, if the strain is applied as an ideal step function [32], the relaxation function given by the PEC model is separable and can be given by

$$
\frac{\tau_{r \theta}}{\gamma_{0}}=G\left(t, \gamma_{0}\right)=\exp (-t) h\left(\gamma_{0}\right),
$$

where the damping function $h(\gamma)$ is given by [29]

$$
h\left(\gamma_{0}\right)=\frac{1}{1+\xi \gamma_{0}^{2} / 3} .
$$

In the PEC+M model, for an ideal step in strain, the relaxation of the second shorter species gives an additional contribution and the response is:

$$
\frac{\tau_{r \theta}}{\gamma_{0}}=G\left(t, \gamma_{0}\right)=\exp (-t) h\left(\gamma_{0}\right)+n_{B} \exp (-t / \epsilon)
$$

where we expect $\epsilon=\lambda_{B} / \lambda_{A}<<1$. Therefore,

$$
\frac{G\left(t, \gamma_{0}\right)}{\exp (-t)}=h\left(\gamma_{0}\right)+n_{B} \exp (t-t / \epsilon) \sim h\left(\gamma_{0}\right) \text { for } t=O(1) \text {. }
$$




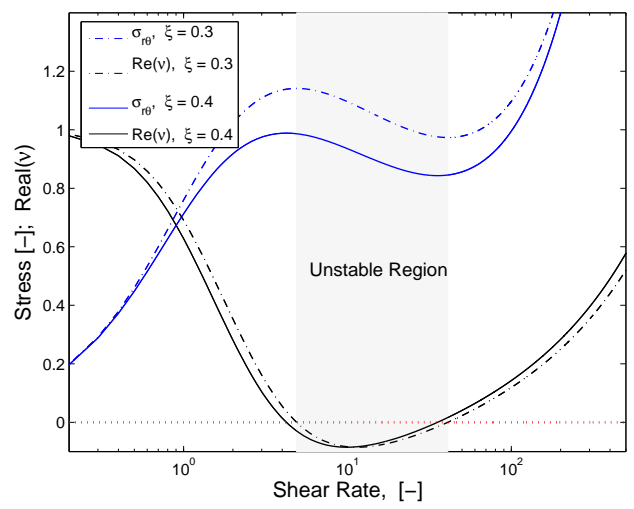

(a)

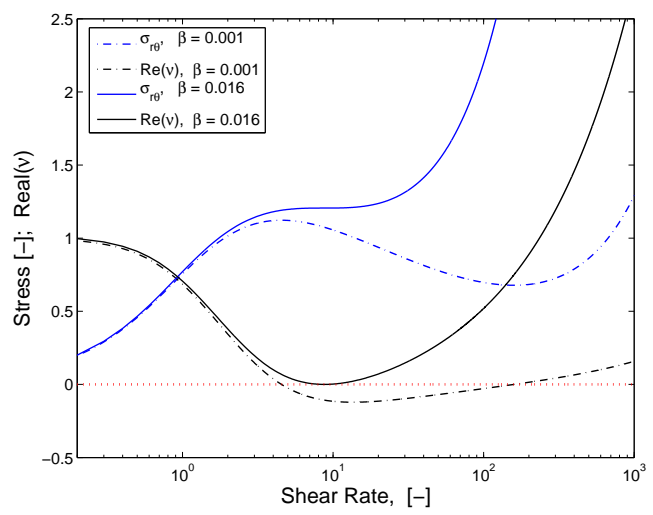

(b)

Figure 4: (a) Steady state shear stress curve (blue) and the the smallest eigenvalue (in black) for two different values of the nonlinear constitutive parameter $\xi$. In this figure $\beta=0.005, f(0.005)=2.19, g(0.005)=18.32$. (b) Steady state shear stress curve and the largest eigenvalue for different values of the solvent viscosity $\beta$. In this figure $\xi=0.3$ and $f(0.001)=2.03, g(0.001)=71.21$.

Note that for the quasilinear species $B$ we can solve analytically for the relaxation function for a ramp in strain given by Equation (29) to obtain

$$
B_{r \theta}=\gamma_{0} n_{B} \frac{b^{2}}{(b-1)^{2}}\left[e^{-t / \epsilon}-e^{-b t / \epsilon}-(b-1) t e^{-b t / \epsilon}\right] .
$$

Thus, for "ideal" steps with $b \rightarrow \infty$, the effect of this species can only be observed at times $t \sim O(\epsilon)$. Typical motor response times may be in the range $10-100 \mathrm{~ms}$ and measuring the relaxation of the second species is thus expected to be convoluted with the motor response function.

\section{Measured versus Calculated Material Func- tions}

As noted in the introduction, because the flow is inhomogeneous there is a difference between the apparent value of any variable and its local value. As defined in equations (3) and (26), the apparent shear rate in dimensionless form is

$$
\dot{\gamma}_{a p p}=\frac{v_{i}}{r_{o}-r_{i}}=D e,
$$

because we selected the gap width as the characteristic length scale [30]. In computing normal stress differences the distinction between apparent and local values becomes even more pronounced since experimental values are not actually 
measured, but rather derived from the total thrust on the wall. For a circular Couette cell this needs some clarification [17].

The $r, \theta$ components of the momentum equation in cylindrical coordinates are:

$$
\begin{array}{ll}
r: & E^{-1} \frac{v^{2}}{r}=\left[\frac{1}{r} \frac{\partial}{\partial r}\left(r \tau_{r r}\right)-\frac{\tau_{\theta \theta}}{r}\right]+\frac{\partial p}{\partial r}, \\
\theta: & E^{-1} \frac{\partial v}{\partial t}=-\frac{1}{r^{2}} \frac{\partial}{\partial r}\left(r^{2} \tau_{r \theta}\right),
\end{array}
$$

where

$$
\begin{aligned}
\boldsymbol{\tau} & =\boldsymbol{\tau}_{s}+\boldsymbol{\tau}_{p} \\
& =-\beta \dot{\gamma}+\left\{-\mathbf{A}-2 \mathbf{B}+\left(1+n_{2}\right) \boldsymbol{I}\right\} .
\end{aligned}
$$

Equation (49) can be rewritten as

$$
E^{-1} \frac{v^{2}}{r}=\frac{\partial p}{\partial r}+\frac{\partial \tau_{r r}}{\partial r}-\frac{\left(\tau_{\theta \theta}-\tau_{r r}\right)}{r},
$$

and ignoring inertial terms $(E>>1)$, we obtain

$$
0=\frac{\partial \Pi_{r r}}{\partial r}-\frac{N_{1}}{r},
$$

where $\Pi_{r r}=\left(p+\tau_{r r}\right)$ is the experimentally measured normal stress or thrust per unit area on the wall of the Couette cell. Hence to compare our calculations with the experimental data we need to find $\Pi_{r r}$ in terms of $N_{1}$. Integrating Equation (53) yields,

$$
\Pi_{r r}\left(r_{i}\right)-\Pi_{r r}\left(r_{o}\right)=\int_{r_{o}}^{r_{i}} \frac{N_{1}}{r} d r .
$$

This allows calculation, from an inhomogeneous distribution $N_{1}(r)$, of the difference in the thrusts at the inner and outer walls. Note that alternatively we could have calculated $\Pi_{r r}$ directly but we would then have had to calculate the unknown pressure $p$ to obtain total stress.

In the limit of small gap size, $\left(r_{o}-r_{i}\right) / r_{i}<<1$, the variation in the shear rate becomes small. In this viscometric limit, $N_{1}$ also varies slowly across the gap and Equation (53) can be used to define the average first normal stress difference, $N_{1}$, as

$$
\frac{N_{1}}{\bar{r}} \equiv \frac{\Pi_{r r}\left(r_{i}\right)-\Pi_{r r}\left(r_{o}\right)}{r_{i}-r_{o}} .
$$

Here $\bar{r}$ is the average gap radius, $\bar{r}=\left(r_{o}+r_{i}\right) / 2$. Thus,

$$
N_{1}=\frac{\bar{r}}{r_{i}-r_{o}}\left[\Pi_{r r}\left(r_{i}\right)-\Pi_{r r}\left(r_{o}\right)\right],
$$

and $N_{1}$ can be determined in a circular Couette device by measuring the difference in the thrusts at the inner and outer walls [17]. As the magnitude of 
the gap increases, the flow becomes increasingly inhomogeneous and the shear stress, shear rate and the first normal stress difference all vary across the gap. We denote the first normal stress difference computed through Equation (56) as $N_{1 \text { app }}$ since it is evaluated assuming that stresses vary smoothly and slowly across the gap. On the other hand our calculated $N_{1}(r)$ depends on the radius and cannot be taken out of the integral in Equation (54). Thus in order to compare potential experimental results with those obtained through numerical calculations we calculate the expression,

$$
N_{1 a p p}=\frac{\bar{r}}{r_{i}-r_{o}}\left[\Pi_{r r}\left(r_{i}\right)-\Pi_{r r}\left(r_{o}\right)\right]=\frac{\bar{r}}{r_{i}-r_{o}} \int_{r_{o}}^{r_{i}} \frac{N_{1}(r)}{r} d r .
$$

\section{Results in Inhomogeneous Flow. PEC and $\mathrm{PEC}+\mathrm{M}$ models}

In viscometric flow the equations to be solved form a coupled system of nonlinear ordinary differential equations in time. For inhomogeneous flow, spatial variations in the velocity gradient result in a non-linear coupled system of partial differential equations. The inhomogeneous system was solved using the CrankNicholson scheme coupled with iterations at each time step on the nonlinear part. The momentum equation is coupled to the stress equations by the presence of the viscous stress $\beta \dot{\gamma}(r)$, and the momentum equation is used to update the velocity at each time step. When the elasticity number, $E$, is finite, that is for flows with finite fluid inertia, the momentum equation is solved together with the stress equations using the Crank-Nicholson scheme which then itself updates the velocity.

The parameter values chosen in this study for the PEC model are $\xi=0.3$ and $\beta=0.005$. The choice of $\xi$ was taken to be close to the best fit to experimental curves for the full VCM two interacting species model in viscometric studies [30]. The value of $\beta$ was chosen to assure that the steady state flow curve for this model is non-monotonic. This value of $\beta$ is smaller than that chosen by Olmsted for the Johnson-Segalman calculations [22], $\beta=0.05$, but still larger than would be anticipated by the experimental results for wormlike micellar solutions, albeit neither model is constructed to agree directly with experiments. For the PEC $+\mathrm{M}$ model we continue with $\xi=0.3$ and use $\epsilon=\lambda_{B} / \lambda_{A}=4.35 \times 10^{-3}$, $n_{B}=1.1331$ so that $\epsilon n_{B}=0.005$, to mimic the solvent viscosity effect in the PEC model. In addition, these values are roughly consistent with experimentally fitted parameters from the full VCM model. In the PEC+M model we use a more "realistic" value for the non-dimensional solvent viscosity, $\beta=6.78 \times 10^{-5}$. The shear stress predictions from the PEC $+\mathrm{M}$ model are anticipated to behave very much like the PEC model at moderate shear rates or step strains since the second species has a relatively short relaxation time, hence its elasticity only becomes important in relatively strong or sudden events. Indeed, numerical solution of the system, for the set of parameters chosen here, shows that in steady simple shear flow the flow curves, i.e. the steady shear stress versus 
shear rate, for the two models are similar although curves for the first normal stress difference differ (see Figure 3).

\subsection{Start up of Steady Shear Flow}

As noted above, in shear flow, the behavior of the shear stress as predicted by both the PEC and PEC+M models is similar; furthermore the time evolution to steady state under shear without diffusion for both models shows dependence on the applied flow history. Figure 5 shows the history-dependent steady state shear stress for the inertialess PEC model as a function of the apparent shear rate, $\dot{\gamma}_{a p p}$ defined in Equation (48). The different curves correspond to the different flow histories noted in Section 3.2, namely jump-up, jump-down, ramp-up and ramp-down. Olmsted et al. [22] demonstrated, for the Johnson-Segalman model, that the geometric curvature $p$ also influenced the flow curves predicted by the model; therefore we carried out a study of the effect of curvature in this paper for the PEC and PEC+M models. Figure 5(a) shows calculations for a curvature of $p=0.1$, Figure 5(b) shows similar results but for a smaller curvature namely $p=0.01$, where the curvature is defined as

$$
p=\frac{r_{o}-r_{i}}{r_{i}}=\frac{1}{r_{i}} .
$$

Typical values for the curvature of circular Couette rheometers range from $p=$ 0.079 [6] to $p=0.13$ [41]. For comparison, calculations with faster start-up, for example, $\tanh (7 t)$, were also computed. The differences for the $\tanh (2 t)$ and $\tanh (7 t)$ for jump down conditions were negligible. For jump up protocols, in the plateau region, the stress curves from the faster jump lies slightly lower at higher shear rates than the slower jump up test. In both cases all curves lie within the envelope of the ramp up and ramp down curves.

When diffusion is added to the PEC, or PEC $+\mathrm{M}$ model, the system selects a unique plateau-like apparent flow curve; that is, the solution is independent of the flow history as shown in Figure 5 by the solid line. The selected "plateau" is fairly flat for $p=0.01$, but has a small positive slope for the larger curvature, $p=0.1$. This general behavior is similar to that of the baseline (JohnsonSegalman) model [22]. Changes in the conformational diffusion constant from $\delta_{A}=0.005$ to $\delta_{A}=0.01$, do not effectively change the plateau of the steady state shear stress, but do slightly decrease the overshoot in the steady state flow curve at the start of the plateau. The presence of a small overshoot in the apparent equilibrium flow curve as well as path-dependent equilibrium flow curves are features commonly observed in experiments with micellar solutions [42-44] and in calculations using the Johnson-Segalman model [22] as well as another "toy" nonlinear model [45].

In Figure 6 we show in greater detail the evolution in the local stress and shear rate within the Couette fixture. In this figure solid symbols represent the values at the inner walls and hollow symbols represent the values at the outer wall. The solid curve is the non-monotonic viscometric prediction as in Figure 1. When the imposed shear rate is within the multivalued region of this curve 


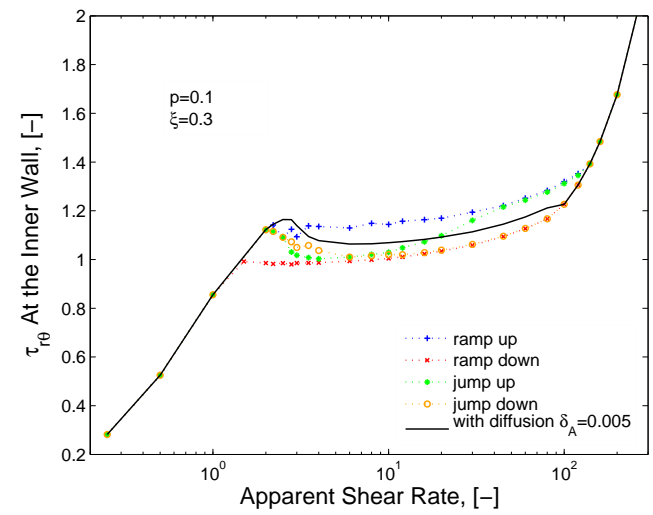

(a)

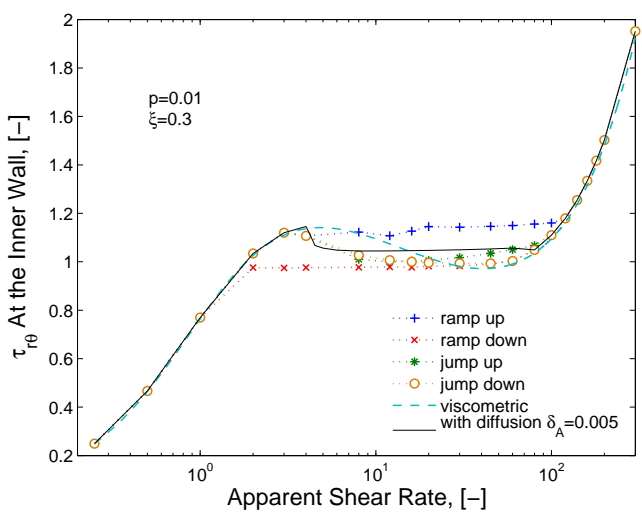

(b)

Figure 5: Steady state flow curves for the PEC model (which are similar to those for the PEC+M model), (a) $p=0.1$ (b) $p=0.01$. The figures show the history dependence for ramp-up: $v_{i}(t)=D e \tanh (0.1 t)$; ramp-down: $v_{i}(t)=$ $300(1-\tanh (0.1 t))+D e \tanh (0.1 t)$; jump-up: $v_{i}(t)=D e \tanh (2 t)$; and jump down: $v_{i}(t)=300(1-\tanh (2 t))+D e \tanh (2 t)$. Here the model parameters are $\delta_{A}=0$, $\xi=0.3, \beta=0.005$. When configuration-dependent diffusion is added we obtain an unique plateau, shown as the solid line in the figures. In this case $\delta_{A}=0.005$. In figure (b), the dash line is the non-monotonic viscometric predictions.

there is one point on the left portion of the curve, corresponding to the shear rate at the outer wall, and one at the right side of the curve corresponding to the shear rate at the inner wall. These points represent the final velocity profiles chosen within the gap, and show "shear banding", with the high shear rate at the inner or moving wall and low shear rate at the outer or fixed wall. The banded structure of the resulting velocity field at $D e=10$ is clearly seen in the inset figure. The two regions represent very different local chain conformation and are connected by a narrow matching region of width $\sqrt{\delta_{A}}$ in which conformationdriven diffusion is important. We also note that the values of the local stress at the inner and outer walls differ slightly in each profile (at $D e=6,10$ or 200) due to the curvature of the geometry. From the momentum equation (Equation (50)) in inertialess flow, $\tau_{r \theta}=c / r^{2}$ where the constant $c=\tau_{\omega} r_{i}^{2}$ is the wall shear stress at the inner cylinder.

Because the shear stress decays monotonically across the gap (by an amount $O(p)$ for narrow gaps), any attempt to reconstruct the underlying nonmonotonic flow curve from local measurements is doomed to failure. To illustrate this, in Figure 7(a) we show the "reconstructed" flow curve that is formed by taking the exact local value of the shear stress and the local value of the shear rate (using the definition in Equation (4)) from the computed velocity profiles. The velocity profiles from these individual numerical experiments are shown in Figure 7(b) in dimensionless form. As the imposed wall velocity exceeds 


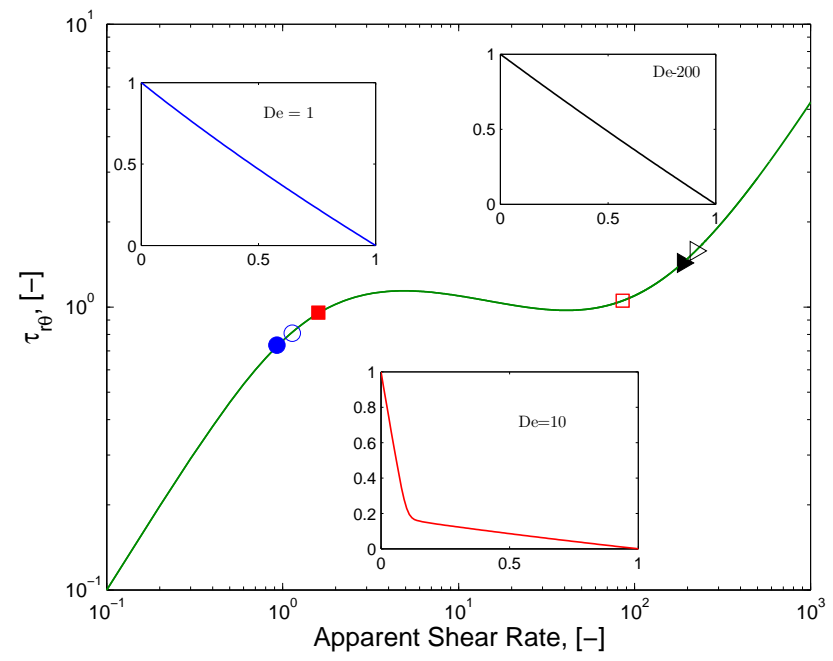

Figure 6: Viscometric flow curve and local values of the shear rate and shear stress for the PEC model. The inserts show the resulting normalized velocity profiles for each apparent shear rate. The values of $D e$ are: 6 ( o: inner wall, •: outer wall), 10 ( $\square$ : inner wall, $\square$ : outer wall), and 200 ( $\triangleleft$ : inner wall, ४: outer wall). In this figure $\beta=0.005, \xi=0.3, \delta_{A}=0.005, p=0.1$, and $E^{-1}=0$.

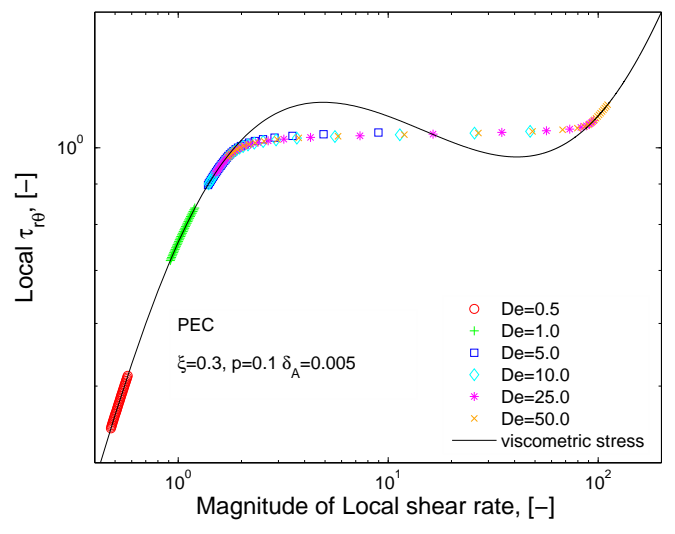

(a)

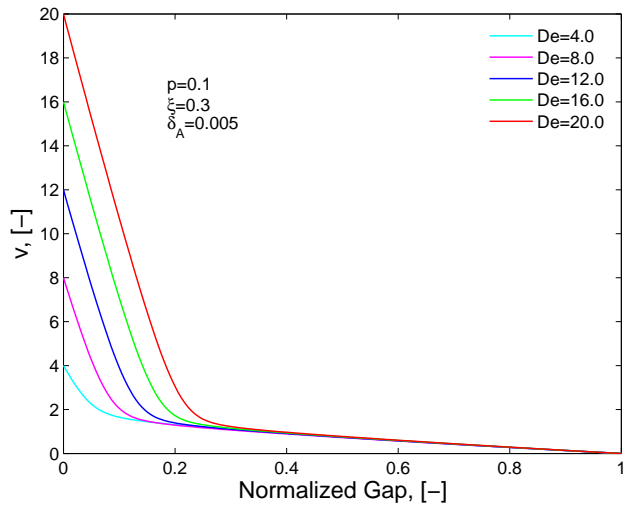

(b)

Figure 7: (a). Reconstructed local shear stress flow curve as a function of local shear rate for the PEC model. (b). Steady state velocity profiles across the gap for the PEC model. In both figures $\beta=0.005, \xi=0.3, \delta_{A}=0.005, p=0.1$, and $E^{-1}=0$.

$D e=2$, an increasingly banded profile is established. It is clear that the reconstructed flow curve in Figure $7(\mathrm{a})$ is monotonic; fitting a power law $\tau_{r \theta}=k \dot{\gamma}^{m}$ 
to the data between $2 \leq \dot{\gamma} \leq 100$ gives a small positive slope of $m=0.016$. This is in clear contrast to the underlying flow curve obtained from an ideal homogeneous flow assumption. In recent experiments with entangled polymer solutions, $\mathrm{Hu}$ et al. argue that observing a locally-monotonic "reconstructed flow curve" is evidence of an underlying monotonic constitutive relation [19]. The present example indicates that such observation of monotonicity cannot be used to make inferences about the underlying homogeneous curve. Even in the limit of $p \rightarrow 0$ the homogeneous solution shown by the solid line in Figure 7(a) is linearly unstable to perturbations in the non-monotonic region and will evolve into a globally monotonic solution consistent with the boundary conditions on velocity and chain conformation at $r=r_{i}, r_{o}$.

Figure 8(a) shows the steady state velocity profiles across the gap for various apparent shear rates. The evolution of the shear bands with respect to apparent shear rate and the gap position is shown in Figure $8(\mathrm{~b})$. While this behavior is similar to that observed by Olmsted et al. [22], it differs in one aspect: in the Johnson-Segalman results, under jump up conditions with $p=0.01$, an inverted band and a two interface band are observed in the plateau region. However, neither the inverted band patterns nor the multiple banding patterns were seen in our computation of the PEC or PEC+M models. A single banded structure with the highest deformation rate at the inner (moving) wall is always obtained.

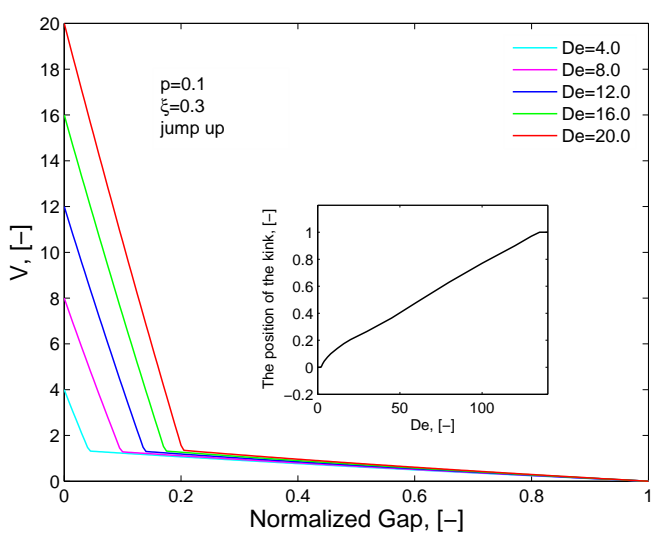

(a)

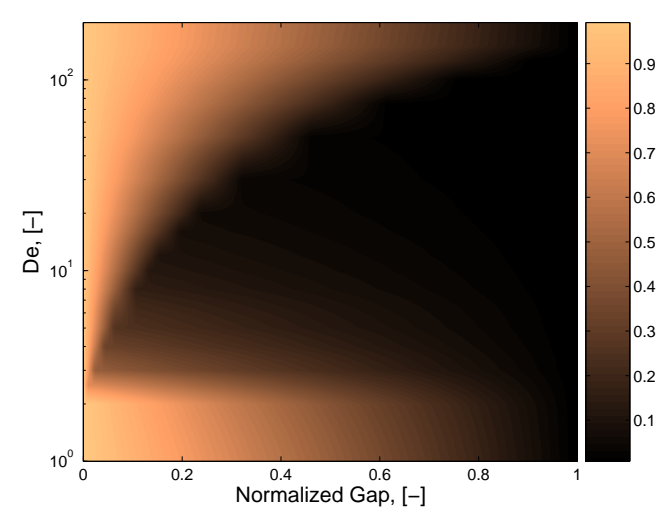

(b)

Figure 8: (a). Steady state velocity profiles across the gap for the PEC model. The insert is the position of the kink as a function of the Deborah number. (b). Evolution of the dimensionless velocity $v(D e) / v_{i}$ as a function of dimensionless position $y$ for different values of apparent shear rate $\dot{\gamma}_{a p p}$, regions where there is a gradual change in color from dark to light correspond to homogeneous velocity profiles, sudden changes in color indicate shear banding. In both figures $\beta=0.005, \xi=0.3, \delta_{A}=0, p=0.1$, and $E^{-1}=0$. Jump up means tanh $2 t$.

The inset in Figure 8(a) shows the percentage of the gap occupied by the high shear rate band, i.e., the distance from the inner cylinder to the "kink" in 
the spatial velocity profile as a function of $D e$. Note that the position $y_{k}(D e)$ varies linearly for Deborah numbers within the plateau region in agreement with experimental observations [8]. A linear regression gives very roughly $y_{k}(D e) \approx$ $D e / 140$. A contour plot representation of the extent of the shear band is shown in Figure 8(b). At low $D e<2$ the velocity varies smoothly across the gap. At higher $D e$, the rapid variation in velocity over a small spatial region is the hallmark of the onset of shear banding. The sharpness of the kink is controlled by the diffusivity parameter $\delta_{A}$, that is there is a smoothing layer of width $\sqrt{\delta_{A}}$ at the kink [30]. Precise experimental values for the conformation-driven diffusivity are not available, but dimensional scaling suggests they should be small. The calculations in Figure $7(\mathrm{a})$ and $7(\mathrm{~b})$ are for $\delta_{A}=0.005$ and show a smooth velocity transition between bands. In Figure $8(\mathrm{a})$ and $8(\mathrm{~b})$ the sharp transitions result from considering the limiting case $\delta_{A} \rightarrow 0$.

The steady state first normal stress difference at the inner wall as well as the apparent first normal stress difference obtained using the formula in Equation (57) for the PEC model, and for the PEC+M model, are shown in Figure 9. As anticipated from the viscometric results in Section 3.3, at small $D e$ before the onset of banding, the curve is quadratic, $N_{1} \sim \dot{\gamma}^{2}$. Since $\dot{\gamma}_{a p p} \approx \dot{\gamma}_{\text {inner }}$ in this region, there is little difference between $N_{1 \text { app }}$ and $N_{1 \text { inner }}$ as expected for an almost homogenous flow. For the range of gap velocities that delineate the shear stress plateau region, the first normal stress difference also shows a banded structure as a consequence of the shear rate bands. Thus to calculate $N_{1 a p p}$ (Equation (57)) for these values of $D e$, the integral must be broken into two pieces:

$$
N_{1 \text { app }}=\bar{r}\left(\int_{r_{i}}^{r_{i}+y_{k}} N_{1}(r ; D e) \frac{1}{r} d r+\int_{r_{i}+y_{k}}^{r_{o}} N_{1}(r ; D e) \frac{1}{r} d r\right)
$$

where $r_{i}+y_{k}(D e)$ is the spatial location of the kink in the velocity profile and is, for the parameter values we've used, $y_{k}(D e) \approx D e / 140$. In the PEC model the normal stress at the inner wall reaches an asymptotic plateau of value $3 / \xi$ given by Equation (39) because the solvent provides no contribution to the normal stress difference. By contrast, the two species PEC $+\mathrm{M}$ model shows an upper quadratic response due to the elastic response of the shorter ' $\mathrm{B}$ ' chains. Profiles of the local distribution in $N_{1}(r ; D e)$ across the gap are shown in Figure 10. For $r<r_{i}+y_{k}$, the normal stress difference asymptotically approaches $3 / \xi$, since the local shear rate is large. For $r>r_{i}+y_{k}$, Figure 10 shows that $N_{1}$ decreases weakly with $r$ and increases roughly linearly with $D e$. For a first estimate of the behavior of $N_{1 a p p}$, we assume that $N_{1}$ in this latter region is independent of $r$. Thus, the integrals in (59) can be evaluated. By exploiting the small value of $y_{k} / r_{i}$, the computed integral can be expressed as a quadratic function of $D e$. Our fits to $N_{1 a p p}$ in the shear stress plateau region show that

$$
N_{1 a p p} \sim 7.5 \times 10^{-2} \mathrm{De}-2.0 \times 10^{-4} \mathrm{De} e^{2}+2.6,
$$

in which the numerical coefficients depend on the specific values of $\xi, p$ and $\beta$. Thus in the plateau region of shear stress, the apparent first normal stress 
difference is a linearly increasing function of $D e$ with a small quadratic correction. As the shear rate continues to climb beyond the shear rates defining the shear stress plateau, $N_{1 \text { app }}$ becomes constant for the PEC model, again as anticipated from the viscometric solution discussed in Section 3.3. The graphs for the PEC+M model differ from those of the PEC model in that for large shear rates the curve returns to quadratic growth as a function of shear rate. This is the result of adding the second non-Newtonian species.

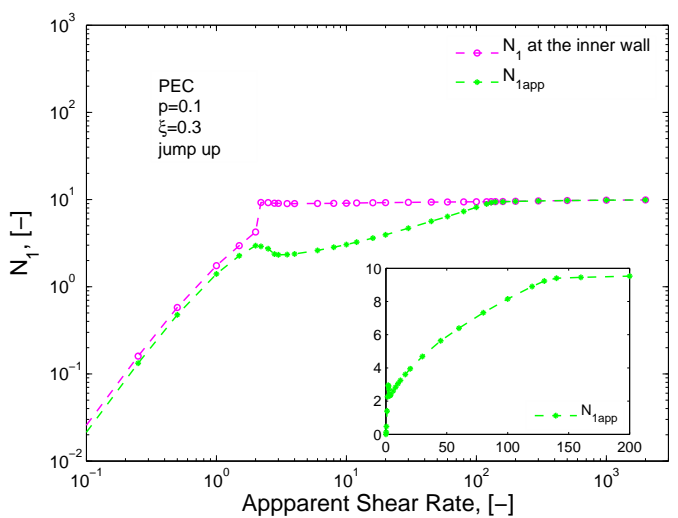

(a)

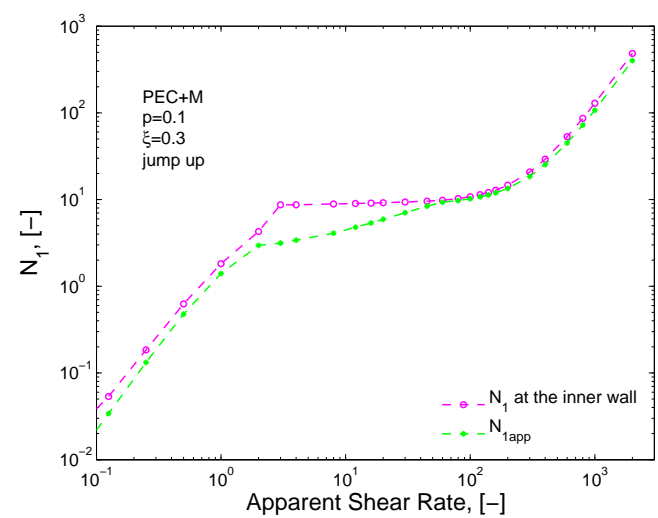

(b)

Figure 9: First normal stress difference as a function of the apparent shear rate for (a) the PEC model, (b) the PEC $+\mathrm{M}$ model. In the PEC model, the parameters are $\delta_{A}=0, \xi=0.3, p=0.1$ and $E^{-1}=0$. In the PEC+M model, the parameters are $\delta_{A}=\delta_{B}=0, \xi=0.3, n_{B}=1.1331, \epsilon=4.35 \times 10^{-3}, p=0.1$ and $E^{-1}=0.02$. The inset in (a) shows the variation in the apparent first normal stress difference $N_{1 a p p}$ on a linear scale as a function of the apparent shear rate in the PEC model.

Figure 11(a) shows the development in time of the shear stress at the inner wall following start up of steady shear flow at a non-dimensional apparent shear rate, $D e=6$. Similarly, Figure 11(b) shows the transient first normal stress difference at the inner wall for the same apparent shear rate. The dashed lines show the equivalent model response if a homogeneous flow is assumed a priori. In these figures we have identified specific points along the curve for comparison purposes with Figures 11(c) and 12. Figure 11(c) shows the velocity profiles across the gap at each of these points and this allows us to compare the evolution of the shear bands in time corresponding to the different regimes observed in both the transient shear stress and first normal stress difference.

In conjunction with the evolution in the kinematics observed in Figure 11(c), we also show in Figure 12 the spatial and temporal development of the longer 'A' species contribution to the shear stress and first normal stress difference in time. In the linear regime at short times $t / \lambda_{A}<<1$, point $\mathrm{A}$, the velocity variation and stress distribution across the gap are approximately homogeneous with variation of $O(p)$. At point $\mathrm{B}$, corresponding to the first overshoot of 


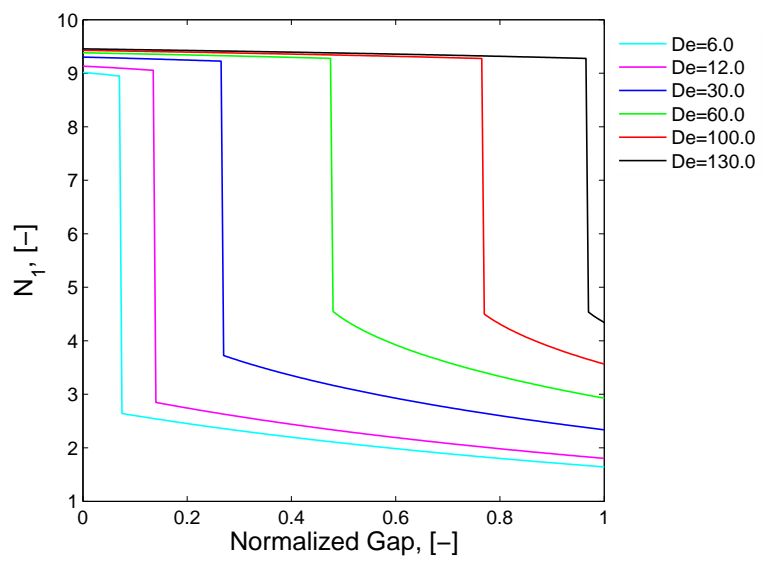

Figure 10: The first normal stress difference of the PEC model across the gap for various $D e$ in the steady state shear stress plateau region after a jump up condition. In this figure $\beta=0.005, \xi=0.3, \delta_{A}=0, p=0.1$.

the shear stress and the onset of non-linearities, a more pronounced deviation from the Newtonian velocity profile is observed. From points C to D, which correspond to the maximum in the curve of the transient first normal stress difference, the maximum deviation of the velocity from the expected linear profile is observed. In this region the velocity curve actually has a small spatial region of negative velocity (although the total integrated displacement of Lagrangian material points is always positive) indicating that the response has overshot and is now settling back. Negative or "recoil" velocities of this type followed by slow approach to ultimate steady banded state have been recently observed in local PIV measurements across the gap in monodisperse polybutadiene solutions [18]. From $\mathrm{E}$ to $\mathrm{F}$ the system is stabilizing towards its steady state and by point $\mathrm{G}$ at $t>>\lambda_{A}$ steady state has been achieved. The steady state velocity profile at this point in Figure 11(c) corresponds to the profile obtained from Figure 8 (b) when a line is drawn horizontally for a Deborah number $D e=6$. Note that as observed experimentally, the high shear rate band region is near the moving wall while the lower shear-rate band is at the fixed wall. In conjunction with these bands in the velocity profiles there are corresponding "bands" in the elastic contributions to the total stress as shown in Figure 12. Interestingly the viscoelastic contribution to the shear stress (Figure 12(a)) decreases near the moving wall, although the monotonic variation in the total stress $\tau_{r \theta}=c / r^{2}$ required by conservation of momentum is still satisfied by the presence of a large viscous contribution to the shear stress in this high shear rate band. The decrease in the elastic contribution to the shear stress in the plateau region following onset of banding is consistent with the entanglement-disentanglement transition hypothesized by Tapadia and Wang $[13,18]$. The normal stress varies 
monotonically across the gap but shows a very sudden saturation in time unlike the normal viscoelastic response predicted by the homogeneous model. This is also consistent with measurements in a monodisperse entangled solution undergoing shear banding [46].

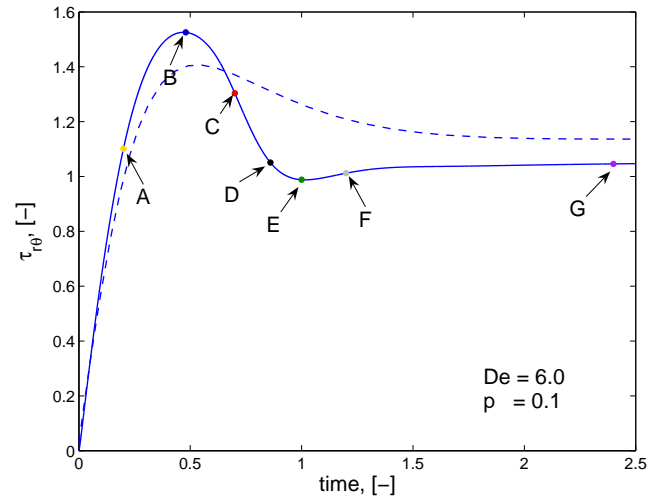

(a)

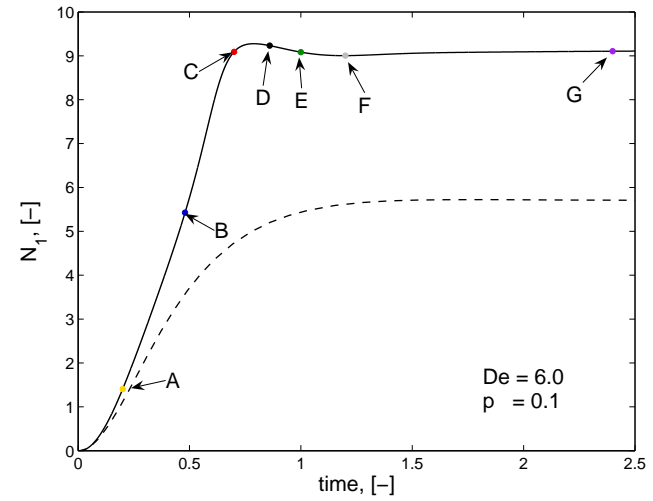

(b)

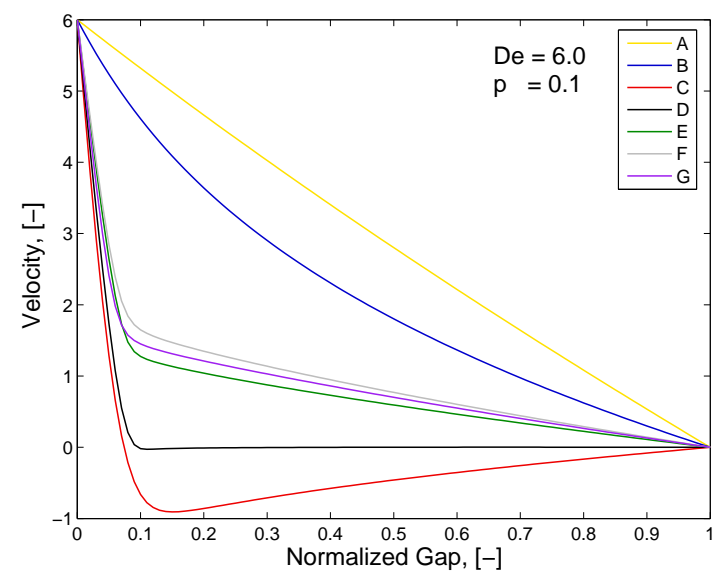

(c)

Figure 11: PEC model predictions in start up of steady shear flow. (a) Shear stress at the inner wall as a function of time. (b) First normal stress difference at the inner wall as a function of time. (c) Velocity profiles across the gap as function of time. In these figures $\beta=0.005, \xi=0.3, \delta_{A}=0.005, p=0.1, E^{-1}=0$, and $v_{i}(t)=6 \tanh (100 t)$. 


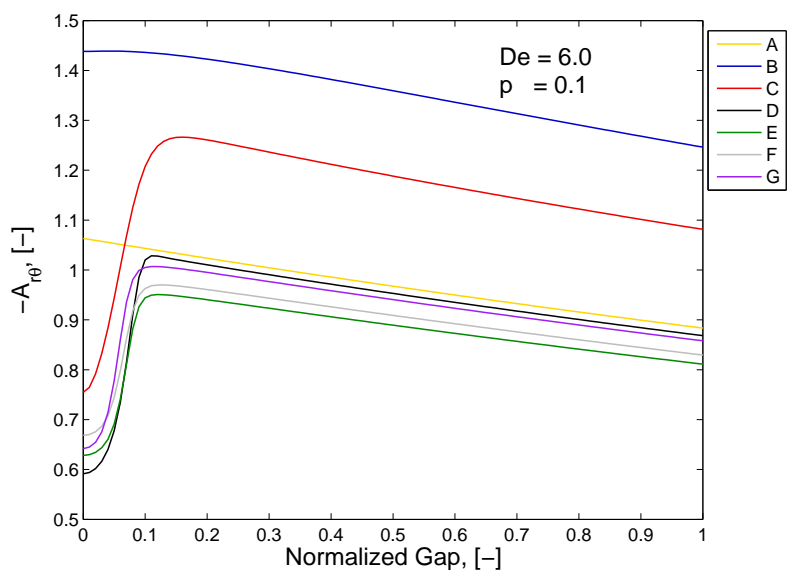

(a)

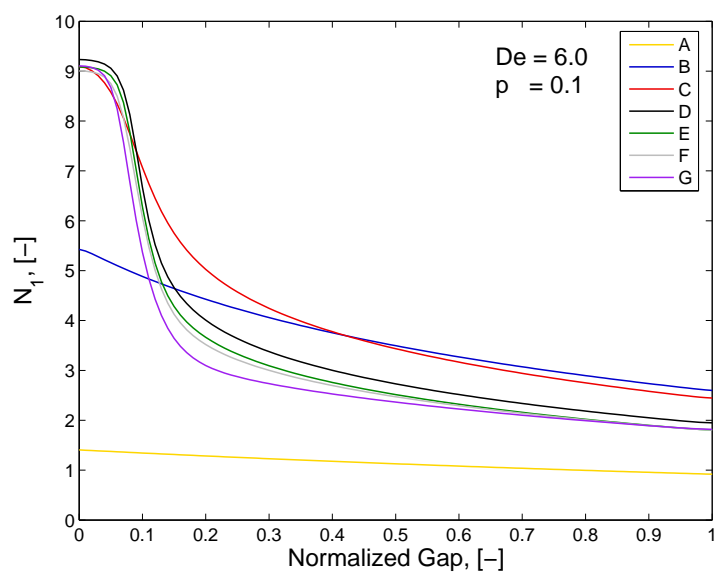

(b)

Figure 12: Shear stress and first normal stress difference across the gap as they develop in time for the PEC model. Note: the different colors in the legend correspond to the times of the points selected in Figure 11(a). In these figures $\beta=0.005, \xi=0.3$, $\delta_{A}=0.005, p=0.1, E^{-1}=0$, and $v_{i}(t)=6 \tanh (100 t)$. 


\subsection{Step Strain}

The stress relaxation predicted by the PEC (or indeed PEC+M) model at the inner cylinder, after a step strain of amplitude $\gamma_{0}$ is shown on a log-log scale in Figure 13. The insert in this figure shows the same computational results but on a semilog scale. The curves in the insert are parallel to each other but vertically shifted, and the inhomogeneous solution for this model shows timestrain separability with a dimensionless decay rate of -1 corresponding to the (dimensional) relaxation time $\lambda_{A}$.

Figure 14 shows the numerically evaluated damping function $h\left(\gamma_{0}\right)$ for the PEC (or PEC+M) model for two different values of the parameter $b$ (defined in Equation (29)) compared with the analytic solution in an "ideal" step strain $(b \rightarrow \infty)$. Close examination of Figure 13 suggests that at high applied strains there is an additional relaxation process at short times, but we know that the PEC model as formulated only has a single relaxation mode (with time constant $\lambda_{A}$ ). Detailed computational investigation shows that this additional relaxation in fact arises from convolution of the viscoelastic relaxation and the finite motor response time required to achieve large strains of $\gamma_{0} \approx 5-10$. As the motor response rate $b$ is increased the curve approaches the theoretical response given by Equation (44). However for any finite $b$ the damping function appears to show two distinct regions. This is consistent with experimental observations in some polymeric entangled systems necessitating a double exponential fit to agree with data [29].

In Figure 15(a) the local strain predicted by the PEC (or PEC+M) model is plotted across the gap after one relaxation time for different $\gamma_{0}$. For small $\gamma_{0}$ the flow is homogenous and the strain is roughly homogeneous with variations of order $\gamma_{0} p$ across the gap. At a critical strain of $\gamma_{0} \approx 2.5$, strain localization occurs with a large gradient of strain developing near the inner cylinder which levels off towards the outer cylinder. The spatial extent of this large strain gradient grows across the gap as the apparent strain increases, although the steepness of the gradient itself decreases. At larger strains, $\gamma_{0} \geq 20$, the gradient in the strain returns to varying approximately linearly across the gap, so the banding structure occurs only for strains $\gamma_{0}$ of roughly 2.5 to 20. Figure 15(b) shows the normalized shear rate at the inner wall as a function of time. Note that for strains in the banding region a second local maximum occurs in the response. The first overshoot comes from the ramp in strain, Equation (29), used to mimic the motor response in step strain experiments, and occurs at $t=1 / b$. Faster ramps would move the overshoot to smaller times, so that if an "ideal" step is applied such an overshoot becomes "infinitely" large at "infinitely" small times. This behavior is an indication that early flow histories imposed by a motor with finite response time cannot be ignored in the analysis of these complex inhomogeneous deformations. The second overshoot, on the other hand, is a signature of the banding phenomenon, since it is only observed at strains which correspond to inhomogeneous profiles across the gap.

Figure 16 shows the strain at the inner cylinder versus the imposed strain $\gamma_{0}$. The onset of the banding behavior corresponds to the sharp rise of this curve, 


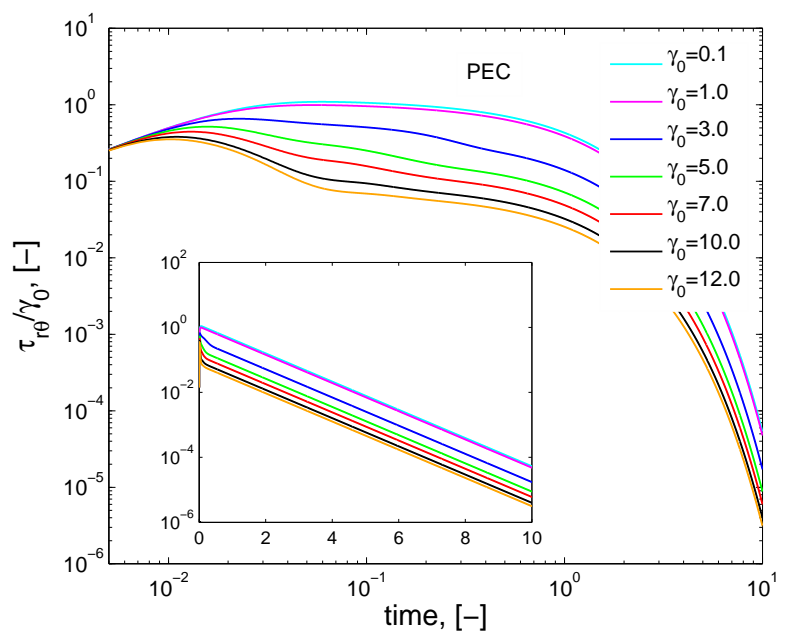

Figure 13: Relaxation moduli of the PEC model here $\xi=0.3, \beta=0.005, p=0.1$, $\delta_{A}=0$ and $E^{-1}=0$. Insert: semi-log plot.

thereafter the region showing the high gradient in the local strain expands across the gap (and decreases in steepness) with increasing imposed strain. The strain rate at the inner cylinder stabilizes and, once banding is complete, the two strains become identical. Figure 16 also shows the direct effect of curvature on the banding. As $p$ decreases, the severity of the shear-banding diminishes.

In Figure 17(a) we show the ratio of the first normal stress difference to the shear stress versus the imposed strain after one relaxation time as predicted by the PEC (or PEC+M) model. The different curves correspond to (1) the LodgeMeissner viscometric expectation, namely $N_{1} / \tau_{r \theta}=\gamma_{0} ;(2)$ the stress ratio at the inner cylinder resulting from a inhomogeneous flow calculation; and (3) the apparent stress ratio resulting from "apparent" or gap-averaged quantities. The Lodge-Meissner rule is uniformly valid up to $\gamma_{0} \sim 2.5$. The stress ratio at the inner cylinder jumps up at $\gamma_{0} \sim 2.5$ as anticipated from the banding profiles observed in Figure 15. Thereafter it levels off until $\gamma_{0}>20$ at which point it merges with the predicted value of the strain (this marks the end of the banding region). The apparent stress curve roughly follows the Lodge-Meissner relation to strains of $\gamma_{0} \sim 20$ for $b=100$, thereafter it falls off below the curve. This is identical to the observed experimental behavior [36].

Figure 17(a) also shows the effect of the motor response rate $b$ on the stress ratio measured in a step strain experiment. For larger $b$ the apparent stress ratios (and those at the inner cylinder after banding) follow the Lodge-Meissner relation to larger strains (for $b=1000$ they extend to $\gamma_{0}=60$ ). Figure $17(\mathrm{a})$ shows that the value of $b$ does not affect the banding region, it only affects the agreement with the Lodge-Meissner relation resulting from the ideal homo- 


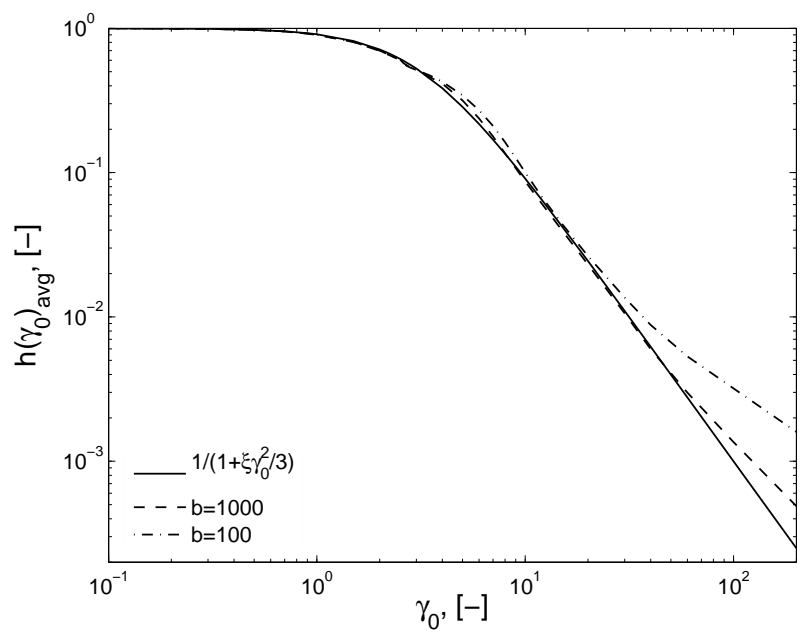

Figure 14: Damping function as a function of $\gamma_{0}$ after one relaxation time for the PEC model. Here, $\xi=0.3, \beta=0.005, p=0.1, \delta_{A}=0$ and $E^{-1}=0$.

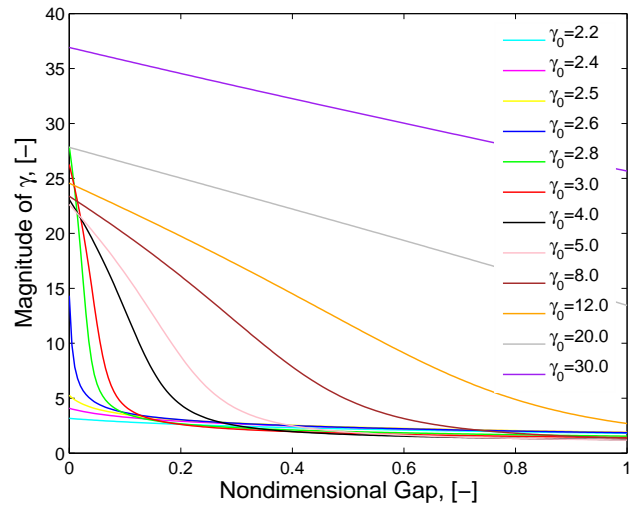

(a)

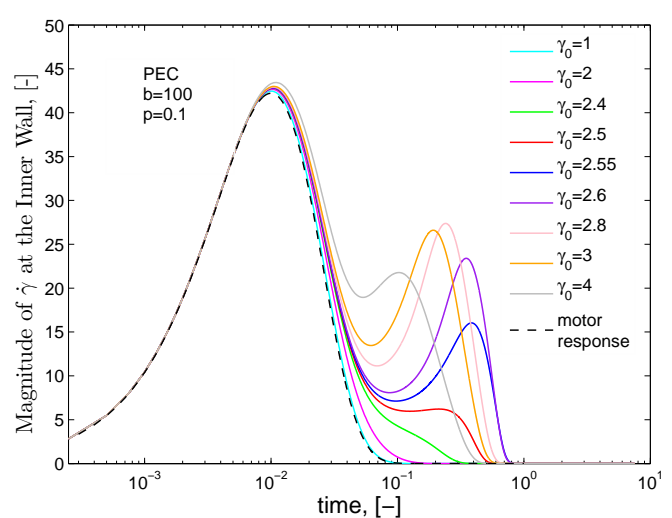

(b)

Figure 15: (a). Magnitude of the local strain $\gamma$ after one effective relaxation time as a function of $r$ following displacement at the inner cylinder, $\gamma_{0}$ for the PEC model. (b). Normalized shear rate at the inner wall as a function of time for the PEC model. In both figures, $\xi=0.3, \beta=0.005, p=0.1, \delta_{A}=0$ and $E^{-1}=0$.

geneous/affine deformation or the value of the Lodge-Meissner ratio evaluated using apparent 'gap-averaged' quantities. Figure 17(b) shows that the curvature parameter $p$ only affects the banding region (as in Figure 16) and not the agreement with the Lodge-Meissner relation. The deviation from the Lodge-Meissner 


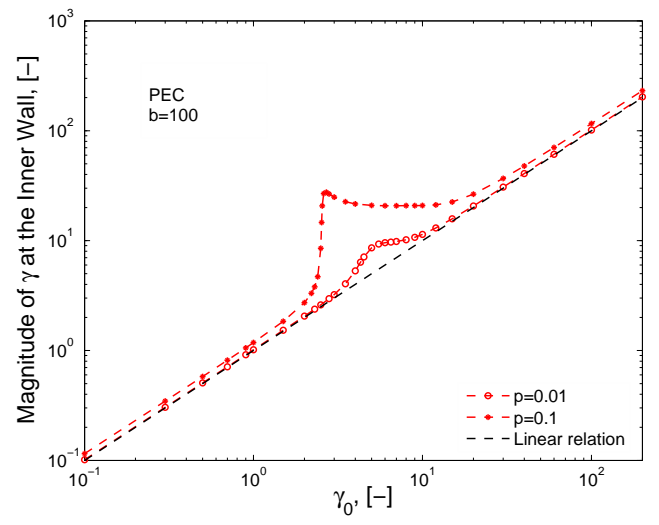

Figure 16: Magnitude of the local strain (at the inner cylinder) versus imposed strain after one effective relaxation time following displacement at the inner cylinder, $\gamma_{0}$. Here, $\xi=0.3, \beta=0.005, b=100, \delta_{A}=0$ and $E^{-1}=0$.

relation observed in Figure 17(b) for large $\gamma_{0}$ is due solely to the imperfect motor response; as $b$ increases, the Lodge-Meissner relation is satisfied to larger and larger applied strains.

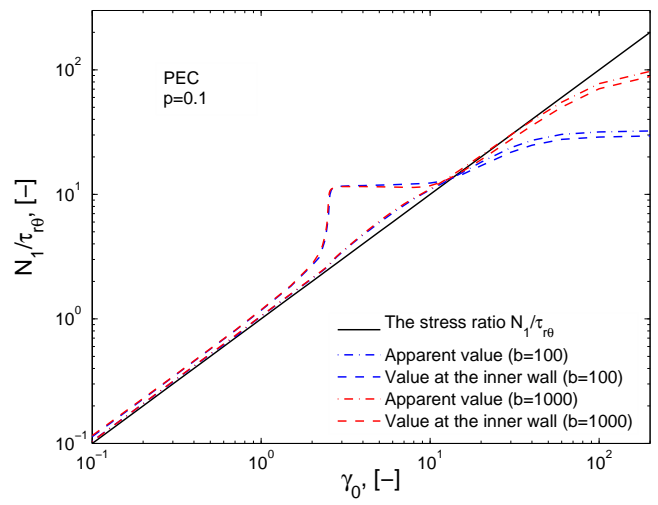

(a)

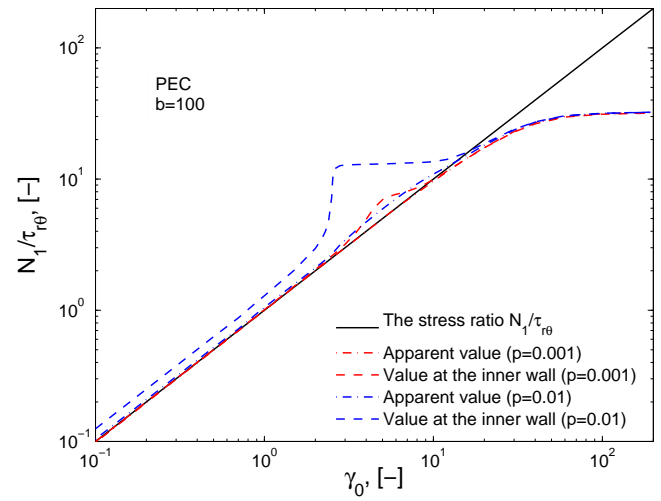

(b)

Figure 17: The stress ratio $N_{1} / \tau_{r \theta}$ as a function of $\gamma_{0}$. (a) For different value of the motor response rate $b$. (b) For different geometric curvature $p$. In these two figures, $\xi=0.3, \beta=0.005, \delta_{A}=0$ and $E^{-1}=0$. 


\section{Conclusion}

In this work we have examined the spatial and temporal evolution of inhomogeneous flow fields in viscometric devices such as cylindrical Couette cells. The basic formulation is that of a two species elastic network model which can capture, in a self-consistent manner, the creation and destruction of elasticallyactive chains as well as the effects of conformational-driven diffusion which arise in areas with large spatial gradients of the local stress or deformation. In the limit of no creation or destruction in the number of each species, the constitutive equations reduce to a two mode non-interacting model that is consistent with the partially extending and convecting (PEC) strand framework proposed by Larson. Computations on this model provide a good qualitative description of recent experimental measurements of inhomogeneous flow development in concentrated solutions of monodisperse entangled polymer chains $[18,19,46]$. It also sets the stage for future study of nonhomogeneous flows of the full (interacting) two species scission/reforming model for wormlike micellar solutions [30].

Our calculations with the single-mode and two-mode PEC type models show that the underlying non-monotonic relationship between shear stress and deformation rate in homogeneous flow leads to the development of shear bands in inhomogeneous flow. The precise shape of the resulting flow curve that is obtained macroscopically depends on the specifics of the experimental procedure (e.g. rapid step increases vs. slow ramps) and test fixture (e.g. the relative magnitude of the gap $\left.p=\left(r_{o}-r_{i}\right) / r_{i}\right)$ as well as the relative magnitude of the local conformation-driven diffusivity parameter $\left(\delta_{A}\right)$. These steady state computational observations are consistent with results from an alternate single species model that has been investigated by Olmsted et al. in inhomogeneous flow [22]. In particular when $\delta_{A} \neq 0$, a unique flow curve results independent of start-up conditions. Time-dependent calculations in 'step strain' experiments and in start up of steady shear flow show that the velocity profile across the gap and the total stress measured at the solid surfaces are coupled and evolve in a complex (and non-monotonic) manner as the shear bands develop. This is consistent with recent time-resolved particle imaging velocimetry measurements in both concentrated polymer solutions [18] and in wormlike micellar solutions [6]. The computational approach developed here can also be extended to more complex deformation histories such as large amplitude oscillatory shear (LAOS) which have recently been investigated experimentally [20].

These computational results have a number of important implications for experimental viscometric observations of 'apparent' or 'gap-averaged' quantities. Firstly, it can be noted that - in contrast to models of Johnson-Segalman type - the present model does not lead to unphysical oscillations in the damping function $h\left(\gamma_{0}\right)$. Experimental measurements of the damping function can thus be used to obtain values of the nonlinear model parameter $(\xi)$ which controls the nonlinear enhancement in the destruction rate of elastically active components. Secondly, in our computational 'step strain' experiments, the coupling between the imposed deformation history and the spatio-temporal development of the 
shear bands results in an apparent departure from the Lodge-Meissner rule at large strains; however the extent of this departure is also convolved with the specifics of the response function of the motor driving the wall displacement. Finally, in steady shear flow, the small but finite spatial inhomogeneity of the global stress field realized in the device (controlled by the magnitude of $p$ ) cannot be neglected.

Our calculations with the both one-species (PEC) and two-species (PEC+M) models show that at steady state for our parameter range the system always selects uniquely a banded structure with the higher deformation rate region near the inner, moving cylinder. This is in contrast to calculations with a JohnsonSeagalman type model [22] which can show reversal in the band ordering, but it is consistent with many experimental observations of local velocity fields in Couette cells. However, this also implies that point-wise reconstruction of the flow curve from local measurements of the velocity gradient and the known stress distribution in the device (see Figures 6 and 7) will always lead to a monotonic curve, albeit of very small slope (depending on the value of $p$ ), even though the underlying constitutive relationship is non-monotonic. Considering the inverse problem, it thus does not seem feasible to use local kinematic observations of a monotonic relationship between shear stress and deformation rate to rule out the possibility of a non-monotonic homogeneous flow curve [19]. As we show in Section 3.4 such homogeneous flow curves will be locally unstable to perturbations and the system will evolve to select a new inhomogeneous profile that is consistent with conservation of mass and linear momentum, the appropriate boundary conditions and the details of the viscoelastic constitutive equation. It is thus critical to develop and explore the rheological predictions of realistic constitutive models that self-consistently couple the non-local evolution of the local microstructure and the global state of stress in the fluid.

\section{Acknowledgments}

The authors thank N.J. Kim, C. Pipe, J. Rothstein and E. Miller for many helpful discussions.

This work was supported by the National Science Foundation under NSF-DMS \#0405931 and DMS \#0406590.

\section{References}

[1] M.E. Cates and S.J. Candau. Statics and dynamics of worm-like surfactant micelles. J. Phys.: Condens. Matter, 2:6869-6892, 1990.

[2] P. Olmsted. Dynamics and flow-induced phase separation in polymeric fluids. Current opinion in colloid and interface science, 4:95-100, 1999.

[3] V.J. Anderson, J.R.A. Pearson, and E.S. Boek. The rheology of worm-like micellar fluids. Rheology Reviews, pages 217-254, 2006. 
[4] M.E. Cates and S.M. Fielding. Rheology of giant micelles. Advances in Physics, 55:799-879, 2006.

[5] J.Y. Lee, G.G. Fuller, N.E. Hudson, and X.F. Yuan. Investigation of shearbanding structure in wormlike micellar solution by point-wise flow-induced birefringence measurements. J. Rheol., 49:537-550, 2005.

[6] E. Miller and J.P. Rothstein. Transient evolution of shear banding in wormlike micelle solutions. J. Non-Newtonian Fluid Mech., 143:22-37, 2007.

[7] Y.T. Hu and A. Lips. Kinetics and mechanism of shear banding in entangled micellar solutions. J. Rheol., 49:1101-1027, 2005.

[8] J.B. Salmon, A. Colin, S. Manneville, and F. Molino. Velocity profiles in shear-banding wormlike micelles. Phys. Rev. Letters., 90:228303-1 228303-4, 2003.

[9] M.W. Liberatore, F. Nettlesheim, N.J. Wagner, and L. Porcar. Spatially resolved small-angle neutron scattering in the 1-2 plane: A study of shear induced phase-separating wormlike micelles. Phys. Rev. E, 73:20504-120504-4, 2006.

[10] R.W. Mair and P.T. Callaghan. Shear flow of wormlike micelles in pipe and cylindrical Couette geometry as studied by nuclear magnetic resonance microscopy. J. Rheol., 41:901-924, 1997.

[11] M. M. Britton, R. W. Mair, R. K. Lambert, and P. T. Callaghan. Transition to shear banding in pipe and couette flow of wormlike micellar solutions. J. Rheol., 43:897-909, 1999.

[12] L. Becu, S. Manneville, and A. Colin. Spatiotemporal dynamics of wormlike micelles under shear. Phys. Rev. Letters, 93(1):018301-1-018301-4, 2004.

[13] P. Tapadia and S-Q. Wang. Yieldlike constitutive transition in shear flow of entangled polymeric fluids. Phys. Rev. Letters, 31(19):198301-1-198301-4, 2003.

[14] G. Porte, J-F. Berret, and J.L. Harden. Inhomogeneous flows of complex fluids: Mechanical instability versus non-equilibrium phase transition. $J$. Phys. II France., 7:459-472, 1997.

[15] J.F. Berret. Transient rheology of wormlike micelles. Langmuir, 13:22272234, 1997.

[16] J. Yerushalmi, S. Katz, and R. Shinnar. The stability of steady shear flows of some viscoelastic fluids. Chemical Eng. Sci., 25:1891-1902, 1970.

[17] C.W. Macosko. Rheology: Principles, Measurements, and Applications. Wiley/VCH, Poughkeepsie, NY, 1994. 
[18] P. Tapadia and S-Q. Wang. Direct visualization of continuous simple shear in non-Newtonian polymeric fluids. Phys. Rev. Letters., 96:016001-1 016001-4, 2006.

[19] Y.T. Hu, L. Wilen, A. Philips, and A. Lips. Is the constitutive equation for entangled polymers monotonic? J. Rheol., 51(2):275-295, 2007.

[20] P. Tapadia, S. Ravindranath, and S-Q. Wang. Banding in entangled polymer fluids under oscillatory shearing. Phys. Rev. Letters, 96:196001-1196001-4, 2006.

[21] F. Bautista, J.F.A. Soltero, J.H. Perez-Lopez, J.E. Puig, and O. Manero. On the shear banding flow of elongated micellar solutions. J. NonNewtonian Fluid Mech., 94:57-66, 2000.

[22] P.D. Olmsted, O. Radulescu, and C.Y.D. Lu. Johnson-Segalman model with a diffusion term in cylindrical Couette flow. J. Rheol., 44:257-275, 2000 .

[23] M. Johnson and D. Segalman. A model for viscoelastic fluid behavior which allows non-affine deformation. J. Non-Newtonian Fluid Mech., 2:255-270, 1977.

[24] L.F. Rossi, G.H. McKinley, and L.P. Cook. Slippage and migration in Taylor-Couette flow of a model for dilute wormlike micellar solutions. $J$. Non-Newtonian Fluid Mech., 136:79-92, 2006.

[25] P. Español, X.F. Yuan, and R.C. Ball. Shear banding flow in the JohnsonSegalman fluid. J. Non-Newtonian Fluid Mech., 65:93-109, 1996.

[26] F. Greco and R.C. Ball. Shear-band formation in a non-Newtonian fluid model with a constitutive instability. J. Non-Newtonian Fluid Mech., 69: 195-206, 1997.

[27] C.Y.D. Lu, P.D. Olmsted, and R.C. Ball. Effects of nonlocal stress on the determination of shear banding flow. Phys. Rev. Letters, 84:642-645, 2000.

[28] M. M. Britton and P. J. Callaghan. Nuclear magnetic resonance visualization of anomalous flow in cone-and-plate rheometry. J. Rheol., 41(6): 1365-1386, 1997.

[29] R.G. Larson. Constitutive Equations for Polymer Melts and Solutions. Butterworths Series in Chemical Engineering, ed. H. Brenner. Butterworths, Boston, 1988.

[30] P.A. Vasquez, L.P. Cook, and G.H. McKinley. A network scission model for wormlike micellar solutions I: Model formulation and homogeneous flow predictions. J. Non-Newtonian Fluid Mech., 144:122-139, 2007. 
[31] M.E. Cates. Reptation of living polymers: Dynamics of entangled polymers in the presence of reversible chain-scission reactions. Macromolecules, 20: 2289-2296, 1987.

[32] R.G. Larson. A constitutive equation for polymer melts based on partially extending strand convection. J. Rheol., 28:545-571, 1984.

[33] R.B. Bird, C.F. Curtiss, R.C. Armstrong, and O. Hassager. Dynamics of Polymeric Liquids: Vol 2, Kinetic Theory. John Wiley and Sons, New York, second edition, 1987.

[34] A.V. Bhave, R.C. Armstrong, and R.A. Brown. Kinetic theory and rheology of dilute, non-homogeneous polymer solutions. J. Chem. Phys., 95:29883000, 1991.

[35] A.N. Beris and V.G. Mavrantzas. On the compatibility between various macroscopic formalisms for the concentration and flow of dilute polymer solutions. J. Rheol., 38:1235-1250, 1994.

[36] C.J. Pipe, N.J. Kim, G.H. McKinley, P.A. Vasquez, and L.P. Cook. Wormlike micellar solutions II: Comparison between experimental data and scission model predictions. In preparation, 2007.

[37] V.G. Mavrantzas and A.N. Beris. Theoretical study of wall effects on rheology of dilute polymer solutions. J. Rheol., 36:175-213, 1992.

[38] W.B. Black and M. D. Graham. Slip, concentration fluctuations, and flow instability in sheared polymer solutions. Macromolecules, 34:5731-5733, 2001.

[39] L.P. Cook and L.F. Rossi. Slippage and migration in models of dilute wormlike micellar solutions and polymeric fluids. J. Non-Newtonian Fluid Mech., 116:347-369, 2004.

[40] P.A. Vasquez. Modeling Wormlike Micellar Solutions. PhD thesis, University of Delaware, 2007.

[41] M.W. Liberatore, F. Nettesheim, E. Kaler, N.J. Wagner, P.A. Vasquez, L.P. Cook, L. Pocar, and Y.T. Hu. Microstructure and shear rheology of entangled wormlike micelles in solution. In Preparation, 2007.

[42] P. Fischer and H. Rehage. Non-linear flow properties of viscoelastic surfactant solutions. Rheol. Acta, 36:13-27, 1997.

[43] C. Grand, J. Arrault, and M.E. Cates. Slow transients and metastability in wormlike micelle rheology. J. Phys. II France, 7:1071-1086, 1997.

[44] A.F. Mendez-Sanchez, M.R. Lopez-Gonzalez, V.H. Rolon-Garrido, J. Perez-Gonzalez, and L. de Vargas. Instabilities of micellar systems under homogeneous and non-homogeneous flow conditions. Rheol. Acta, 42: $56-63,2003$. 
[45] N.A. Spenley, X.F. Yuan, and M.E. Cates. Nonmonotonic constitutive laws and the formation of shear-banded flows. J.Phys. II France, 6:551-571, 1996.

[46] P. Tapadia and S-Q. Wang. Nonlinear flow behavior of entangled polymer solutions: yield like entanglement-disentanglement transition. Macromolecules, 37:9083-9095, 2004. 